\title{
Asymptotic Analysis of the Ponzano-Regge Model with Non-Commutative Metric Boundary Data
}

\author{
Daniele $\mathrm{ORITI}^{\dagger}$ and Matti RAASAKKA ${ }^{\ddagger}$ \\ $\dagger$ Max Planck Institute for Gravitational Physics (Albert Einstein Institute), \\ Am Mühlenberg 1, 14476 Potsdam, Germany \\ E-mail: daniele.oriti@aei.mpg.de \\ $\ddagger$ LIPN, Institut Galilée, CNRS UMR 7030, Université Paris 13, Sorbonne Paris Cité, \\ 99 av. Clement, 93430 Villetaneuse, France \\ E-mail: matti.raasakka@lipn.univ-paris13.fr
}

Received February 04, 2014, in final form June 14, 2014; Published online June 26, 2014

http://dx.doi.org/10.3842/SIGMA.2014.067

\begin{abstract}
We apply the non-commutative Fourier transform for Lie groups to formulate the non-commutative metric representation of the Ponzano-Regge spin foam model for $3 \mathrm{~d}$ quantum gravity. The non-commutative representation allows to express the amplitudes of the model as a first order phase space path integral, whose properties we consider. In particular, we study the asymptotic behavior of the path integral in the semi-classical limit. First, we compare the stationary phase equations in the classical limit for three different noncommutative structures corresponding to the symmetric, Duflo and Freidel-Livine-Majid quantization maps. We find that in order to unambiguously recover discrete geometric constraints for non-commutative metric boundary data through the stationary phase method, the deformation structure of the phase space must be accounted for in the variational calculus. When this is understood, our results demonstrate that the non-commutative metric representation facilitates a convenient semi-classical analysis of the Ponzano-Regge model, which yields as the dominant contribution to the amplitude the cosine of the Regge action in agreement with previous studies. We also consider the asymptotics of the $\mathrm{SU}(2) 6 j$-symbol using the non-commutative phase space path integral for the Ponzano-Regge model, and explain the connection of our results to the previous asymptotic results in terms of coherent states.
\end{abstract}

Key words: Ponzano-Regge model; non-commutative representation; asymptotic analysis

2010 Mathematics Subject Classification: 83C45; 81R60; 83C27; 83C80; 81S10; 53D55

\section{Introduction}

Spin foam models have in recent years arisen to prominence as a possible candidate formulation for the quantum theory of spacetime geometry (see [48] for a thorough review). Their formalism derives mainly from topological quantum field theories [2], Loop Quantum Gravity [55, 60] and discrete gravity, e.g., Regge calculus [51]. On the other hand, spin foam models may also be seen as a generalization of matrix models for $2 \mathrm{~d}$ quantum gravity via group field theory [22, 44]. For $3 \mathrm{~d}$ quantum gravity, the relation between spin foam models and canonical quantum gravity has been fully cleared up. In particular, it is known that the Turaev-Viro model [61] is the covariant version of the canonical quantization (à la Witten [53, 62]) of 3d Riemannian gravity with a positive cosmological constant, while the Ponzano-Regge model is the limit of the former for a vanishing cosmological constant $[1,40,41]$ (see also [42, 43, 50] on incorporating the

${ }^{\star}$ This paper is a contribution to the Special Issue on Deformations of Space-Time and its Symmetries. The full collection is available at http://www.emis.de/journals/SIGMA/space-time.html 
cosmological constant in $3 \mathrm{~d}$ LQG and $[56,57]$ for further work on relating $3 \mathrm{~d}$ gravity to ChernSimons theory and quantum group structures). In this case, the spin foam 2-complexes have been rigorously shown to arise as histories of LQG spin network states, as initially suggested in [52], while the correspondence between LQG states and the Ponzano-Regge boundary data had been already noted in [54]. However, in $4 \mathrm{~d}$ the situation is less clear. Several different spin foam models for $4 \mathrm{~d}$ Riemannian quantum gravity have been proposed in the literature, such as the Barrett-Crane model [7, 9], the Freidel-Krasnov model [23], a model based on the flux representation [8], and one based on the spinor representation [19], while in the Lorentzian case the Engle-Pereira-Rovelli-Livine model $[20,21]$ represents essentially the state of the art (see also [47] for a review of the new 4d models). These $4 \mathrm{~d}$ models differ specifically in their implementation of the necessary simplicity constraints on the underlying topological BF theory, which should impose geometricity of the 2-complex corresponding to a discrete spacetime manifold and give rise to local degrees of freedom. Thus, a further study of the geometric content of the different spin foam models is certainly welcome. In particular, one might hope to recover discrete Regge gravity in the classical limit of the model, since this would imply an acceptable imposition of the geometric constraints at least in the classical regime. Moreover, classical general relativity can be obtained from the Regge gravity by further taking the continuum limit, which allows for some confidence that continuum general relativity may be recovered also from the continuum limit of the full quantum spin foam model. The Regge action is indeed known to arise as the stationary phase solution in the $3 \mathrm{~d}$ case in the large-spin limit for handlebodies $[17,36]$. In 4d, Regge action was recovered asymptotically first for a single 4-simplex [10] and later for an arbitrary triangulation with a fixed spin labeling, when both boundary and bulk spin variables are scaled to infinity [15, 28, 30, 31, 32]. Recently, in [33, 34], an asymptotic analysis of the full $4 \mathrm{~d}$ partition function was given using microlocal analysis, which revealed some worrying accidental curvature constraints on the geometry of several widely studied $4 \mathrm{~d}$ models. This work considered only the strict asymptotic regime of the spin variables, without further scalings of the parameters of the theory. The work of [29, 38] on the other hand dealt with the large-spin asymptotics of the EPRL model considering also scaling in the Barbero-Immirzi parameter, with interesting results. In particular, the analysis of [29] used also the discrete curvature as an expansion parameter and identified an intermediate regime of large spin values (dependent on the Barbero-Immirzi parameter) that seems to lead to the right Regge behavior of the amplitudes in the small curvature approximation.

Classically, spin foam models, as discretizations of continuum theories, are based on a phase space structure, which is a direct product of cotangent bundles over a Lie group that is the structure group of the corresponding continuum principal bundle (e.g., SU(2) for 3d Riemannian gravity $)^{1}$. The group part of the product of cotangent bundles thus corresponds to discrete connection variables on a triangulated spatial hypersurface, while the cotangent spaces correspond to discrete metric variables (e.g., edge vectors in $3 \mathrm{~d}$, or face bivectors in $4 \mathrm{~d}$, which correspond to discrete tetrad variables due to the simplicity constraints). Accordingly, the geometric data of the classical discretized model is transparently encoded in the cotangent space variables. However, when one goes on to quantize the system to obtain the spin foam model, the cotangent space variables get quantized to differential operators on the group. Typically (for compact Lie groups), these geometric operators possess discrete spectra, and so the transparent classical discrete geometry described by continuous metric variables gets replaced by the

\footnotetext{
${ }^{1}$ In this paper, we are concerned exclusively with the case of topological spin foam models with vanishing cosmological constant. For non-topological models, such as 4d quantum gravity models, the physical configuration space is a homogeneous subspace (or, including the Barbero-Immirzi parameter, a more general subspace) of a Lie group, instead of a Lie group. Likewise, for a non-vanishing cosmological constant, the configuration space is a quantum group. Therefore, in these cases the structure of the physical phase space is, strictly speaking, more involved than what is implied above.
} 
quantum geometry described by discrete spin labels. This corresponds to a representation of the states and amplitudes of the model in terms of eigenstates of the geometric operators, the spin representation - hence the name 'spin' foams. The quantum discreteness of geometric variables in spin foams, i.e., the use of quantum numbers as opposed to phase space variables, although very useful to make contact with the canonical quantum theory, makes the amplitudes lose a direct contact with the classical discrete action and the classical discrete geometric variables. The use of such classical discrete geometric variables, on the other hand, has been prevented until recently by their non-commutative nature.

However, recently, a new mathematical tool was introduced in the context of $3 \mathrm{~d}$ quantum gravity, which became to be called the 'group Fourier transform' [4, 5, 6, 7, 8, 16, 24, 25, 35, 45]. This is an $L^{2}$-isometric map from functions on a Lie group to functions on the cotangent space equipped with a (generically) non-commutative $\star$-product structure. In [27], the transform was generalized to the 'non-commutative Fourier transform' for all exponential Lie groups by deriving it from the canonical symplectic structure of the cotangent bundle, and the non-commutative structure was seen to arise from the deformation quantization of the algebra of geometric operators. Accordingly, the non-commutative but continuous metric variables obtained through the non-commutative Fourier transform correspond to the classical metric variables in the sense of deformation quantization. Thus, it enables one to describe the quantum geometry of spin foam models and group field theory [5, 6] (and Loop Quantum Gravity [4, 16]) by classical-like continuous metric variables.

The aim of this paper is to initiate the application of the above results in analysing the geometric properties of spin foam models, in particular, in the classical limit $(\hbar \rightarrow 0)$. We will restrict our consideration to the 3d Ponzano-Regge model $[11,12,49]$ to have a better control over the formalism in this simpler case. However, already for the Ponzano-Regge model we discover nontrivial properties of the metric representation related to the non-commutative structure, which elucidate aspects of the use of non-commutative Fourier transform in the context of spin foam models. In particular, we find that in applying the stationary phase approximation one must account for the deformation structure of the phase space in the variational calculus in order to recover the correct geometric constraints for the metric variables in the classical limit of the phase space path integral. Otherwise, the classical geometric interpretation of metric boundary data depends on the ambiguous choice of quantization map for the algebra of geometric operators, which seems problematic. Nevertheless, once the deformed variational principle adapted to the non-commutative structure of the phase space is employed, the non-commutative Fourier transform is seen to facilitate an unambiguous and straightforward asymptotic analysis of the full partition function via a non-commutative stationary phase approximation.

In Section 2 we will first outline the formalism of non-commutative Fourier transform, adapted from [27] to the context of gravitational models. In Section 3 we introduce the PonzanoRegge model, seen as a discretization of the continuum 3d BF theory. In Section 4 we then apply the non-commutative Fourier transform to the Ponzano-Regge model to obtain a representation of the model in terms of non-commutative metric variables, and write down an explicit expression for the quantum amplitude for fixed metric boundary data on a boundary with trivial topology. In Section 5 we further study the classical limit of the Ponzano-Regge amplitudes for fixed metric boundary data, and find that the results differ for different choices of noncommutative structures unless one accounts for the deformation structure in the variational calculus. When this is taken into account, the resulting semi-classical approximation coincides with what one expects from a discrete gravity path integral. In particular, if one considers only the partial saddle point approximation obtained by varying the discrete connection only, one finds that the discrete path integral reduces to the one for 2nd order Regge action in terms of discrete triad variables. In Section 6 we consider in more detail the Ponzano-Regge amplitude with non-commutative metric boundary data for a single tetrahedron. We recover the Regge 
action in the classical limit of the amplitude, and explain the connection of our calculation to the previous studies of spin foam asymptotics in terms of coherent states. Section 7 summarizes the obtained results and points to further research.

\section{Non-commutative Fourier transform for $\mathrm{SU}(2)$}

Our exposition of the non-commutative Fourier transform for SU(2) in this section follows [27], adapted to the needs of quantum gravity models. Originally, a specific realization of the noncommutative Fourier transform formalism for the group SO(3) was introduced in [24] by Freidel \& Livine, and later expanded on by Freidel \& Majid [25] and Joung, Mourad \& Noui [35] to the case of $\mathrm{SU}(2)$. (More abstract formulations of a similar concept have appeared also in $[39,58]$.) In our formalism this original version of the transform corresponds to a specific choice of a quantization of the algebra of geometric operators, which we will refer to as the Freidel-Livine-Majid quantization map, and treat it as one of the concrete examples we give of the more general formulation in Subsection 5.1.2

Let us consider the group $\mathrm{SU}(2)$, the Lie algebra $\mathrm{Lie}(\mathrm{SU}(2))=: \mathfrak{s u}(2)$ of $\mathrm{SU}(2)$, and the associated cotangent bundle $\mathcal{T}^{*} \mathrm{SU}(2) \cong \mathrm{SU}(2) \times \mathfrak{s u}(2)^{*}$. As it is a cotangent bundle, $\mathcal{T}^{*} \mathrm{SU}(2)$ carries a canonical symplectic structure. This is given by the Poisson brackets

$$
\left\{O, O^{\prime}\right\} \equiv \frac{\partial O}{\partial X_{i}} \tilde{\mathcal{L}}_{i} O^{\prime}-\tilde{\mathcal{L}}_{i} O \frac{\partial O^{\prime}}{\partial X_{i}}+\lambda \epsilon_{i j}{ }^{k} \frac{\partial O}{\partial X_{i}} \frac{\partial O^{\prime}}{\partial X_{j}} X_{k},
$$

where $O, O^{\prime} \in C^{\infty}\left(\mathcal{T}^{*} \mathrm{SU}(2)\right)$ are classical observables, and $\tilde{\mathcal{L}}_{i}:=\lambda \mathcal{L}_{i}$ are dimensionful Lie derivatives on the group with respect to a basis of right-invariant vector fields. $\lambda \in \mathbb{R}_{+}$is a parameter with dimensions $\left[\frac{\hbar}{X}\right]$, which determines the physical scale associated to the group manifold via the dimensionful Lie derivatives and the structure constants $\left[\tilde{\mathcal{L}}_{i}, \tilde{\mathcal{L}}_{j}\right]=\lambda \epsilon_{i j}{ }^{k} \tilde{\mathcal{L}}_{k}$. $X_{i}$ are the Cartesian coordinates on $\mathfrak{s u}(2)^{*} .^{3}$

Let us now introduce coordinates $\zeta: \mathrm{SU}(2) \backslash\{-e\} \rightarrow \mathfrak{s u}(2) \cong \mathbb{R}^{3}$ on the dense subset $\mathrm{SU}(2) \backslash\{-e\}=: H \subset \mathrm{SU}(2)$, where $e \in \mathrm{SU}(2)$ is the identity element, which satisfy $\zeta(e)=0$ and $\tilde{\mathcal{L}}_{i} \zeta^{j}(e)=\delta_{i}^{j}$. The use of coordinates $\zeta$ on $H$ can be seen as a sort of 'one-point-decompactification' of $\mathrm{SU}(2)$. We then have for the Poisson brackets of the coordinates ${ }^{4}$

$$
\left\{\zeta^{i}, \zeta^{j}\right\}=0, \quad\left\{X_{i}, \zeta^{j}\right\}=\tilde{\mathcal{L}}_{i} \zeta^{j}, \quad\left\{X_{i}, X_{j}\right\}=\lambda \epsilon_{i j}{ }^{k} X_{k}
$$

The Poisson brackets involving $\zeta^{i}$ are, of course, well-defined only on $H$. We see that the commutators $\left\{X_{i}, \zeta^{j}\right\}$ of the chosen canonical variables are generically deformed due to the curvature of the group manifold. They coincide with the usual flat commutation relations associated with Poisson-commuting coordinates only at the identity. Moreover, let us define the deformed addition $\oplus_{\zeta}$ for these coordinates in the neighborhood of identity as $\zeta(g h)=$ : $\zeta(g) \oplus_{\zeta} \zeta(h)$. It holds $\zeta(g) \oplus_{\zeta} \zeta(h)=\zeta(g)+\zeta(h)+\mathcal{O}\left(\lambda^{0},|\ln (g)|,|\ln (h)|\right)$ for any choice of $\zeta$ complying with the above mentioned assumptions. Indeed, the parametrization is chosen so that in the limit $\lambda \rightarrow 0$, while keeping the coordinates $\zeta$ fixed, we effectively recover the flat phase space $\mathcal{T}^{*} \mathbb{R}^{3}=\mathbb{R}^{3} \times \mathbb{R}^{3} \cong \mathfrak{s u}(2) \times \mathfrak{s u}(2)^{*}$ from $\mathcal{T}^{*} \mathrm{SU}(2)=\mathrm{SU}(2) \times \mathfrak{s u}(2)^{*}$. This follows because

\footnotetext{
${ }^{2}$ In addition, another realization of the non-commutative Fourier transform for $\mathrm{SU}(2)$ relying on spinors was formulated by Dupuis, Girelli \& Livine in [18], but we will not consider it here.

${ }^{3}$ Here it seems we are giving dimensions to coordinates, which is usually a bad idea in a gravitational theory, to be considered below. The point here is that the coordinates $X_{i}$ turn out to have a geometric interpretation as discrete triad variables, which is exactly what one would like to give dimensions to in general relativity.

${ }^{4}$ Strictly speaking, the coordinates are not observables of the classical system, but we may consider them defined implicitly, since any observable may be parametrized in terms of them, and they may be approximated arbitrarily closely by classical observables.
} 
keeping $\zeta$ fixed implies a simultaneous scaling of the class angles $|\ln (g)|$ of the group elements. Accordingly, the group effectively coincides with the tangent space $\mathfrak{s u}(2)$ at the identity in this limit, and $\zeta$ become the Euclidean Poisson-commuting coordinates on $\mathfrak{s u}(2) \cong \mathbb{R}^{3}$ for any initial choice of $\zeta$ satisfying the above assumptions. Thus, $\lambda$ can also be thought of as a deformation parameter already at the level of the classical phase space. For the above reasons, we will call the limit $\lambda \rightarrow 0$ the abelian limit.

Let us then consider the quantization of the Poisson algebra given by the Poisson bracket (2.1). In particular, we consider the algebra $\mathfrak{H}$ generated by the operators $\hat{\zeta}^{i}$ and $\hat{X}_{i}$, modulo the commutation relations

$$
\left[\hat{\zeta}^{i}, \hat{\zeta}^{j}\right]=0, \quad\left[\hat{X}_{i}, \hat{\zeta}^{j}\right]=i \hbar \widehat{\tilde{\mathcal{L}}_{i} \zeta^{j}}, \quad\left[\hat{X}_{i}, \hat{X}_{j}\right]=i \hbar \lambda \epsilon_{i j}{ }^{k} \hat{X}_{k} .
$$

These relations follow from the symplectic structure of $\mathcal{T}^{*} \mathrm{SU}(2)$ in the usual way by imposing the relation $\left[\mathfrak{Q}(O), \mathfrak{Q}\left(O^{\prime}\right)\right] \stackrel{!}{=} i \hbar \mathfrak{Q}\left(\left\{O, O^{\prime}\right\}\right)$ with the Poisson brackets of the canonical variables, where by $\mathfrak{Q}: C^{\infty}\left(\mathcal{T}^{*} \mathrm{SU}(2)\right) \rightarrow \mathfrak{H}$ we denote the quantization map specified by linearity, the ordering of operators, and $\mathfrak{Q}\left(\zeta^{i}\right)=: \hat{\zeta}^{i}, \mathfrak{Q}\left(X_{i}\right)=: \hat{X}_{i}$.

We wish to represent the abstract algebra $\mathfrak{H}$ defined by the commutation relations $(2.2)$ as operators acting on a Hilbert space. There exists the canonical representation in terms of smooth functions on $H \subset \mathrm{SU}(2)$ with the $L^{2}$-inner product

$$
\left\langle\psi \mid \psi^{\prime}\right\rangle:=\frac{1}{\lambda^{3}} \int_{H} \mathrm{~d} g \overline{\psi(g)} \psi^{\prime}(g),
$$

where $\mathrm{d} g$ is the normalized Haar measure, and the action of the canonical operators on is given by

$$
\hat{\zeta}^{i} \psi \equiv \zeta^{i} \psi, \quad \hat{X}_{i} \psi \equiv i \hbar \tilde{\mathcal{L}}_{i} \psi
$$

However, we would like to represent our original configuration space $\mathrm{SU}(2)$ rather than $H$, and therefore we will instead consider smooth functions on $\mathrm{SU}(2)$, whose restriction on $H$ is clearly always in $C^{\infty}(H)$. Since the coordinates are well-defined only on $\mathcal{H}=\mathrm{SU}(2) \backslash\{-e\}$, the action of the coordinate operators should then be understood only in a weak sense: Even though strictly speaking the action $\hat{\zeta}^{i} \psi \equiv \zeta^{i} \psi$ is not well-defined for the whole of $\mathrm{SU}(2)$, the inner products $\left\langle\psi\left|\hat{\zeta}^{i}\right| \psi^{\prime}\right\rangle$ are, since we may write

$$
\left\langle\psi\left|\hat{\zeta}^{i}\right| \psi^{\prime}\right\rangle=\frac{1}{\lambda^{3}} \int_{\mathrm{SU}(2)} \mathrm{d} g \overline{\psi(g)} \zeta^{i}(g) \psi^{\prime}(g) \equiv \frac{1}{\lambda^{3}} \int_{H} \mathrm{~d} g \overline{\psi(g)} \zeta^{i}(g) \psi^{\prime}(g)
$$

for smooth $\psi, \psi^{\prime}$. It is easy to verify that the commutation relations are represented correctly with this definition of the action, and the function space may be completed in the $L^{2}$-norm as usual.

However, there is also a representation in terms of another function space, which is obtained through a deformation quantization procedure applied to the operator algebra corresponding to the other factor of the cotangent bundle, $\mathfrak{s u}(2)^{*}$ (see [27] for a thorough exposition). Notice that the restriction of $\mathfrak{H}$ to the subalgebra generated by the operators $\hat{X}_{i}$ is isomorphic to a completion of the universal enveloping algebra $\overline{U(\mathfrak{s u}(2))}$ of $\mathrm{SU}(2)$ due to its Lie algebra commutation relations. A $\star$-product for functions on $\mathfrak{s u}(2)^{*}$ is uniquely specified by the restriction of the quantization map $\mathfrak{Q}$ on the $\mathfrak{s u}(2)^{*}$ part of the phase space via the relation $f \star f^{\prime}:=\mathfrak{Q}^{-1}\left(\mathfrak{Q}(f) \mathfrak{Q}\left(f^{\prime}\right)\right)$, where $f, f^{\prime} \in C^{\infty}\left(\mathfrak{s u}(2)^{*}\right)$ and accordingly $\mathfrak{Q}(f), \mathfrak{Q}\left(f^{\prime}\right) \in \overline{U(\mathfrak{s u}(2))}$. One may verify that the following action of the algebra on functions $\tilde{\psi} \in L_{\star}^{2}\left(\mathfrak{s u}(2)^{*}\right)$ constitutes another representation of the algebra:

$$
\hat{\zeta}^{i} \tilde{\psi} \equiv-i \hbar \frac{\partial \tilde{\psi}}{\partial X_{i}}, \quad \hat{X}_{i} \tilde{\psi} \equiv X_{i} \star \tilde{\psi}
$$


The non-commutative Fourier transform acts as an intertwiner between the canonical representation in terms of square-integrable functions on $\mathrm{SU}(2)$ and the non-commutative dual space $L_{\star}^{2}\left(\mathfrak{s u}(2)^{*}\right)$ of square-integrable functions on $\mathfrak{s u}(2)^{*}$ with respect to the $\star$-product. It is given by

$$
\tilde{\psi}(X) \equiv \int_{H} \frac{\mathrm{d} g}{\lambda^{3}} \overline{E(g, X)} \psi(g) \in L_{\star}^{2}\left(\mathfrak{s u}(2)^{*}\right), \quad \psi \in L^{2}(\mathrm{SU}(2)),
$$

where the integral kernel

$$
E(g, X) \equiv e_{\star}^{\frac{i}{\hbar \lambda} k(g) \cdot X}:=\sum_{n=0}^{\infty} \frac{1}{n !}\left(\frac{i}{\hbar \lambda}\right)^{n} k^{i_{1}}(g) \cdots k^{i_{n}}(g) X_{i_{1}} \star \cdots \star X_{i_{n}}
$$

is the non-commutative plane wave, and we denote $k(g):=-i \ln (g) \in \mathfrak{s u}(2)$ taken in the principal branch of the logarithm. The inverse transform reads

$$
\psi(g)=\int_{\mathfrak{s u}(2)^{*}} \frac{\mathrm{d} X}{(2 \pi \hbar)^{3}} E(g, X) \star \tilde{\psi}(X) \in L^{2}(\mathrm{SU}(2)), \quad \tilde{\psi} \in L_{\star}^{2}\left(\mathfrak{s u}(2)^{*}\right),
$$

where $\mathrm{d} X:=\mathrm{d} X_{1} \mathrm{~d} X_{2} \mathrm{~d} X_{3}$ denotes the Lebesgue measure on the Lie algebra dual $\mathfrak{s u}(2)^{*} \cong \mathbb{R}^{3}$.

Let us list some important properties of the non-commutative plane waves that we will use in the following:

$$
\begin{aligned}
& E(g, X)=e_{\star}^{\frac{i}{\hbar \lambda} k(g) \cdot X} \equiv c(g) e^{\frac{i}{\hbar} \zeta(g) \cdot X}, \quad \text { where } \quad c(g):=E(g, 0), \\
& \overline{E(g, X)}=E\left(g^{-1}, X\right)=E(g,-X), \\
& E\left(\operatorname{ad}_{h} g, X\right)=E\left(g, \operatorname{Ad}_{h}^{-1} X\right), \\
& E(g h, X)=E(g, X) \star E(h, X), \\
& \int_{\mathfrak{s u}(2)^{*}} \frac{\mathrm{d} X}{(2 \pi \hbar \lambda)^{3}} E(g, X)=\delta(g), \\
& \tilde{\psi}(X) \star E(g, X)=E(g, X) \star \tilde{\psi}\left(\operatorname{Ad}_{g} X\right),
\end{aligned}
$$

where $\operatorname{ad}_{h} g:=h g h^{-1}$ and $\operatorname{Ad}_{h} X:=h X h^{-1}$. Notice that from (2.3) and (2.4) it follows that $c\left(\operatorname{ad}_{h} g\right)=c(g)$ and $\zeta\left(\operatorname{ad}_{h} g\right)=h \zeta(g) h^{-1}=: \operatorname{Ad}_{h} \zeta(g)$. In addition, we find that the function

$$
\delta_{\star}(X, Y):=\int_{H} \frac{\mathrm{d} g}{(2 \pi \hbar \lambda)^{3}} \overline{E(g, X)} E(g, Y)
$$

acts as the delta distribution with respect to the $\star$-product, namely,

$$
\int_{\mathfrak{s u}(2)^{*}} \mathrm{~d} Y \delta_{\star}(X, Y) \star \tilde{\psi}(Y)=\tilde{\psi}(X)=\int_{\mathfrak{s u}(2)^{*}} \mathrm{~d} Y \tilde{\psi}(Y) \star \delta_{\star}(X, Y) .
$$

More generally, $\delta_{\star}$ is the integral kernel of the projection

$$
\mathcal{P}(\tilde{\psi})(X):=\int_{\mathfrak{s u}(2)^{*}} \mathrm{~d} Y \delta_{\star}(X, Y) \star \tilde{\psi}(Y)
$$

onto the image $L_{\star}^{2}\left(\mathfrak{s u}(2)^{*}\right)$ of the non-commutative Fourier transform. In the following, we will also occasionally slightly abuse notation by writing

$$
\delta_{\star}\left(\sum_{i} X_{i}\right):=\int_{H} \frac{\mathrm{d} g}{(2 \pi \hbar \lambda)^{3}} \prod_{i} E\left(g, X_{i}\right)
$$


for convenience, although this is not a function of the linear sum $\sum_{i} X_{i}$ if $c(g) \neq 1$ for some $g \in H$.

Finally, we wish to emphasize that the non-commutative coordinate variables of the dual representation are unambiguously identified with the corresponding classical conjugate momenta to the group elements via deformation quantization. This follows directly from the construction. Indeed, it is a key advantage of the above construction for the non-commutative representation that it retains a direct relation to the classical phase space quantities, thus helping to make the interpretation of the quantum expressions more intuitive and straightforward, especially in the semi-classical regime. Our primary goal in this paper is exactly to use this clear-cut relation to our benefit in analysing and interpreting in discrete geometric terms the leading order semi-classical behavior of the Ponzano-Regge model.

\section{3d BF theory and the Ponzano-Regge model}

The Ponzano-Regge model can be understood as a discretization of 3-dimensional Riemannian BF theory. In this section, we will briefly review how it can be derived from the continuum BF theory, while keeping track of the dimensionful physical constants determining the various asymptotic limits of the theory.

Let $\mathcal{M}$ be a 3 -dimensional base manifold to a frame bundle with the structure group $\mathrm{SU}(2)$. Then the partition function of $3 \mathrm{~d}$ BF theory on $\mathcal{M}$ is given by

$$
\mathcal{Z}_{\mathrm{BF}}^{\mathcal{M}}=\int \mathcal{D} E \mathcal{D} \omega \exp \left(\frac{i}{2 \hbar \kappa} \int_{\mathcal{M}} \operatorname{tr}(E \wedge F(\omega))\right)
$$

where $E$ is an $\mathfrak{s u}(2)^{*}$-valued triad 1 -form on $\mathcal{M}, F(\omega)$ is the $\mathfrak{s u}(2)$-valued curvature 2-form associated to the connection 1 -form $\omega$, and the trace is taken in the fundamental spin- $\frac{1}{2}$ representation of $\mathrm{SU}(2) . \hbar$ is the reduced Planck constant and $\kappa$ is a constant with dimensions of inverse momentum. The connection with Riemannian gravity in three spacetime dimensions gives $\kappa:=8 \pi G$, where $G$ is the gravitational constant. Since the triad 1-form $E$ has dimensions of length and the curvature 2 -form $F$ is dimensionless, the exponential is rendered dimensionless by dividing with $\hbar \kappa \equiv 8 \pi l_{p}, l_{p} \equiv \hbar G$ being the Planck length in three dimensions. Integrating over the triad field in (3.1), we get heuristically

$$
\mathcal{Z}_{\mathrm{BF}}^{\mathcal{M}} \propto \int \mathcal{D} \omega \delta(F(\omega))
$$

so we see that the BF partition function is (at least nominally) nothing but the volume of the moduli space of flat connections on $\mathcal{M} .^{5}$ Generically, this is of course divergent, which (among other things) motivates us to consider discretizations of the theory. However, since BF theory is purely topological, that is, it does not depend on the metric structure of the base manifold, such a discretization should not affect its essential properties.

Now, to discretize the continuum BF theory, we first choose a triangulation $\Delta$ of the manifold $\mathcal{M}$, that is, a (homogeneous) simplicial complex homotopic to $\mathcal{M}$. The dual complex $\Delta^{*}$ of $\Delta$ is obtained by replacing each $d$-simplex in $\Delta$ by a $(3-d)$-simplex and retaining the connective relations between simplices. Then, the homotopy between $\Delta$ and $\mathcal{M}$ allows us to think of $\Delta$, and thus $\Delta^{*}$, as embedded in $\mathcal{M}$. We further form a finer cellular complex $\Gamma$ by diving the tetrahedra in $\Delta$ along the faces of $\Delta^{*}$. In particular, $\Gamma$ then consists of tetrahedra $t \in \Delta$, with vertices $t^{*} \in \Delta^{*}$ at their centers, each subdivided into four cubic cells. Moreover,

\footnotetext{
${ }^{5}$ The volume of a moduli space can be defined via its natural symplectic structure, and in some 2-dimensional cases has been rigorously related to a QFT partition function, see [26, 59, 63].
} 


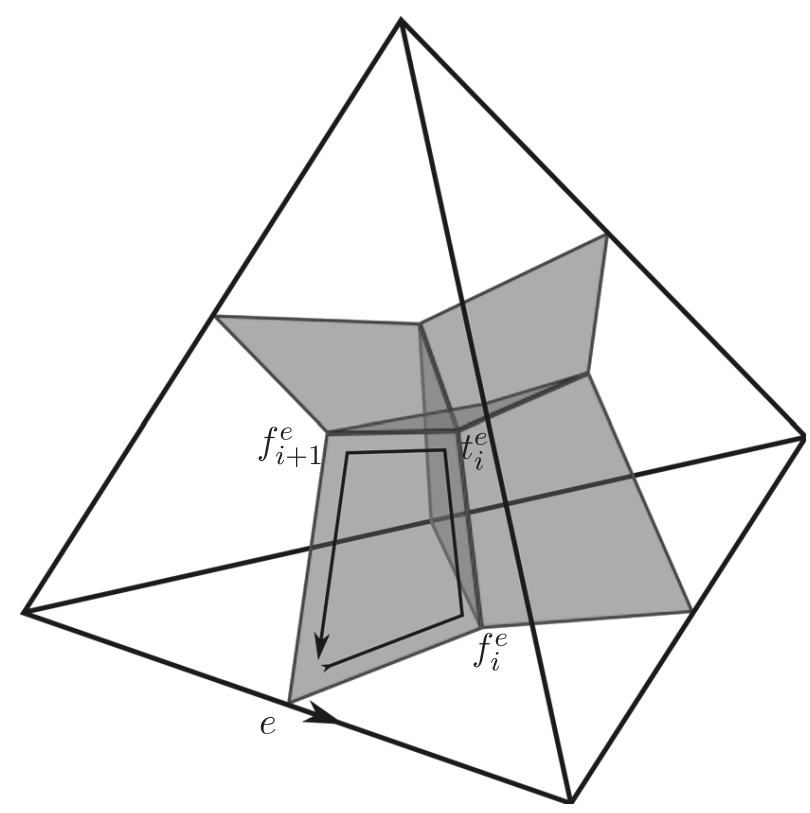

Figure 1. The subdivision of tetrahedra in $\Delta$ into a finer cellular complex $\Gamma$. Here, $e$ labels an edge of the triangulation, $f_{i}^{e}$ label (the centers of) the triangles incident to the edge $e$, and $t_{i}^{e}$ label (the centers of) the tetrahedra incident to $e$. The index $i$ runs from 0 to $n_{e}-1, n_{e}$ being the number of triangles incident to $e$.

for each tetrahedron $t \in \Delta$, there are edges $t f \in \Gamma$, which correspond to half-edges of $f^{*} \in \Delta^{*}$, going from the centers of the triangles $f \in \Delta$ bounding the tetrahedron to the center of the tetrahedron $t$. Also, for each triangle $f \in \Delta$, there are edges ef $\in \Gamma$, which go from the center of the triangle $f \in \Delta$ to the centers of the edges $e \in \Delta$ bounding the triangle $f$. See Fig. 1 for an illustration of the subdivision of a single tetrahedron in $\Delta$.

To obtain the discretized connection variables associated to the triangulation $\Delta$, we integrate the connection along the edges $t f \in \Gamma$ and $e f \in \Gamma$ as

$$
g_{t f}:=\mathcal{P} e^{i \int_{t f} \omega} \in \mathrm{SU}(2) \quad \text { and } \quad g_{e f}:=\mathcal{P} e^{i \int_{e f} \omega} \in \mathrm{SU}(2),
$$

where $\mathcal{P}$ denotes the path-ordered exponential. Thus, they are the Wilson line variables of the connection $\omega$ associated to the edges or, equivalently, the parallel transports from the initial to the final points of the edges with respect to $\omega$. We assume the triangulation $\Delta$ to be piece-wise flat, and associate frames to all simplices of $\Delta$. We then interpret $g_{t f}$ as the group element relating the frame of $t \in \Delta$ to the frame of $f \in \Delta$, and similarly $g_{e f}$ as the group element relating the frame of $f \in \Delta$ to the frame of $e \in \Delta$. Furthermore, we integrate the triad field along the edges $e \in \Delta$ as

$$
X_{e}:=\int_{e} \operatorname{Ad}_{G_{e}} E \in \mathfrak{s u}(2)^{*} .
$$

Here, $G_{e}$ denotes the $\mathrm{SU}(2)$-valued function on the edge $e$ that parallel transports via adjoint action the pointwise values of $E$ along $e$ to a fixed base point at the center of $e$. An orientation for the edge $e$ may be chosen arbitrarily. $X_{e}$ is interpreted as the vector representing the magnitude and the direction of the edge $e$ in the frame associated to the edge $e$ itself.

In the case that $\Delta$ has no boundary, a discrete version of the BF partition function (3.2), the Ponzano-Regge partition function, may be written as

$$
\mathcal{Z}_{\mathrm{PR}}^{\Delta}=\int\left[\prod_{t f} \mathrm{~d} g_{t f}\right] \prod_{e \in \Delta} \delta\left(H_{e^{*}}\left(g_{t f}\right)\right),
$$


where $H_{e^{*}}\left(g_{t f}\right) \in \mathrm{SU}(2)$ are holonomies around the dual faces $e^{*} \in \Delta^{*}$ obtained as products of $g_{t f}, f^{*} \in \partial e^{*}$, and $\mathrm{d} g_{t f}$ is again the Haar measure on $\mathrm{SU}(2)$. Mimicking the continuum partition function of BF theory, the Ponzano-Regge partition function is thus an integral over the flat discrete connections, the delta functions $\delta\left(H_{e^{*}}\left(g_{t f}\right)\right)$ constraining holonomies around all dual faces to be trivial.

Now, we can apply the non-commutative Fourier transform to expand the delta functions in terms of non-commutative plane waves by equation (2.6). This yields

$$
\mathcal{Z}_{\mathrm{PR}}^{\Delta}=\int\left[\prod_{t f} \mathrm{~d} g_{t f}\right]\left[\prod_{e} \frac{\mathrm{d} X_{e}}{(2 \pi \hbar \lambda)^{3}}\right]\left[\prod_{e \in \Delta} c\left(H_{e^{*}}\left(g_{t f}\right)\right)\right] \exp \left\{\frac{i}{\hbar} \sum_{e \in \Delta} X_{e} \cdot \zeta\left(H_{e^{*}}\left(g_{t f}\right)\right)\right\} .
$$

Comparing with (3.1), this expression has a straightforward interpretation as a discretization of the first order path integral of the continuum BF theory. We can clearly identify the discretized triad variables $X_{e}$ in (3.3) with the non-commutative metric variables defined via the noncommutative Fourier transform. We also see that, from the point of view of discretization, the form of the plane waves and thus the choice for the quantization map is directly related to the choice of the precise form for the discretized action and the path integral measure. In particular, the coordinate function $\zeta: \mathrm{SU}(2) \rightarrow \mathfrak{s u}(2)$ and the prefactor $c: \mathrm{SU}(2) \rightarrow \mathbb{C}$ of the noncommutative plane wave are dictated by the choice of the quantization map, and the coordinates specify the discretization prescription for the curvature 2-form $F(\omega)$. Similar interplay between *-product quantization and discretization is well-known in the case of the first order phase space path integral formulation of ordinary quantum mechanics [14]. Moreover, on dimensional grounds, we must identify $\lambda \equiv \kappa=8 \pi G$, so that the coordinates $\zeta$ have the dimensions of $\frac{1}{\kappa} F(\omega)$. Therefore, the abelian limit of the non-commutative structure of the phase space corresponds in this case also physically to the no-gravity limit $G \rightarrow 0$. We will denote this classical deformation parameter associated with the non-commutative structure of the phase space collectively by $\kappa$ in the following.

\section{Non-commutative metric representation of the Ponzano-Regge model}

If the triangulated manifold $\Delta$ has a non-trivial boundary, we may assign connection data on the boundary by fixing the group elements $g_{e f}$ associated to the boundary triangles $f \in \partial \Delta$. Then, the (non-normalized) Ponzano-Regge amplitude for the boundary can be written as

$$
\mathcal{A}_{\mathrm{PR}}\left(g_{e f} \mid f \in \partial \Delta\right)=\int\left[\prod_{t f} \mathrm{~d} g_{t f}\right]\left[\prod_{\substack{e f \\ f \notin \partial \Delta}} \mathrm{d} g_{e f}\right] \prod_{e \in \Delta} \prod_{i=0}^{n_{e}-1} \delta\left(g_{e f_{i+1}^{e}} g_{t_{i}^{e}}^{-1} f_{i+1}^{e} g_{t_{i}^{e}} f_{i}^{e} g_{e f_{i}^{e}}^{-1}\right) .
$$

The delta functions are over the holonomies around the wedges of the triangulation pictured in grey in Fig. 1. For this purpose, the tetrahedra $t_{i}^{e}$ and the triangles $f_{i}^{e}$ sharing the edge $e$ are labelled by an index $i=0, \ldots, n_{e}-1$ in a right-handed fashion with respect to the orientation of the edge $e$ and with the identification $f_{n_{e}} \equiv f_{0}$, as in Fig. 1 .

Let us introduce some simplifying notation. We will choose an arbitrary spanning tree of the dual graph to the boundary triangulation, pick an arbitrary root vertex for the tree, and label the boundary triangles $f_{i} \in \partial \Delta$ by $i \in \mathbb{N}_{0}$ in a compatible way with respect to the partial ordering induced by the tree, so that the root has the label 0 (see Fig. 2). Moreover, we denote the set of ordered pairs of labels associated to neighboring boundary triangles by $\mathcal{N}$, and label the group elements associated to the pair of neighboring boundary triangles $(i, j) \in \mathcal{N}$ as illustrated in Fig. 2. The group elements $g_{t f}, f \notin \partial \Delta$, will be denoted by a collective label $h_{l}$. As we integrate 

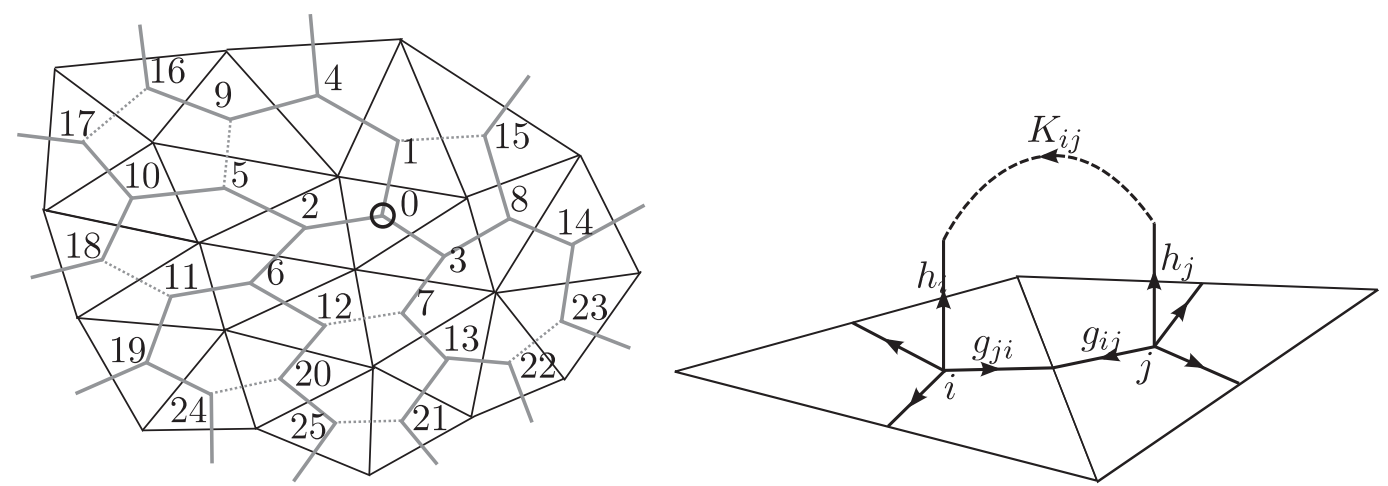

Figure 2. On the left: A portion of a rooted labelled spanning tree of the dual graph of a boundary triangulation (solid grey edges). On the right: Boundary triangles $f_{i}, f_{j} \in \partial \Delta$ and the associated group elements.

over $g_{e f}$ for $f \notin \partial \Delta$ in (4.1), we obtain

$$
\mathcal{A}_{\mathrm{PR}}\left(g_{i j}\right)=\int\left[\prod_{l} \mathrm{~d} h_{l}\right]\left[\prod_{e \notin \partial \Delta} \delta\left(H_{e^{*}}\left(h_{l}\right)\right)\right]\left[\prod_{\substack{(i, j) \in \mathcal{N} \\ i<j}} \delta\left(g_{i j} h_{j}^{-1} K_{j i}\left(h_{l}\right) h_{i} g_{j i}^{-1}\right)\right] .
$$

Here $h_{i}$ is the group element associated to the dual half-edge going from the boundary triangle $i$ to the center of the tetrahedron with triangle $i$ on its boundary, and $K_{i j}\left(h_{l}\right)$ is the holonomy along the bulk dual edges from the center of the tetrahedron with triangle $j$ to the center of the tetrahedron with triangle $i$ (see Fig. 2 for illustration). There is a one-to-one correspondence between the pairs $(i, j)$ of neighboring boundary triangles and faces of the dual 2-complex touching the boundary. Notice that we have chosen here as the base point of each holonomy the boundary dual vertex with a smaller label. By expanding the delta distributions in (4.2) with boundary group variables into non-commutative plane waves, we get

$$
\begin{aligned}
\mathcal{A}_{\mathrm{PR}}\left(g_{i j}\right)= & \int\left[\prod_{l} \mathrm{~d} h_{l}\right]\left[\prod_{e \notin \partial \Delta} \delta\left(H_{e^{*}}\left(h_{l}\right)\right)\right] \\
& \times\left[\prod_{\substack{i, j) \in \mathcal{N} \\
i<j}} \int \frac{\mathrm{d} Y_{j i}}{(2 \pi \hbar \kappa)^{3}} E\left(g_{i j} h_{j}^{-1} K_{j i}\left(h_{l}\right) h_{i} g_{j i}^{-1}, Y_{j i}\right)\right] .
\end{aligned}
$$

To obtain the expression for metric boundary data, we employ the non-commutative Fourier transform,

$$
\tilde{\mathcal{A}}_{\mathrm{PR}}\left(X_{i j}\right)=\int\left[\prod_{(i, j) \in \mathcal{N}} \frac{\mathrm{d} g_{i j}}{\kappa^{3}}\right] \mathcal{A}_{\mathrm{PR}}\left(g_{i j}\right) \prod_{(i, j) \in \mathcal{N}} E\left(g_{i j}^{-1}, X_{i j}\right) .
$$

Here the variable $X_{i j}$ is understood geometrically as the edge vector shared by the triangles $i, j$ as seen from the frame of reference of the triangle $j$. We note that the exact functional form of the amplitude, as that of the non-commutative plane wave, depends on the particular choice of a quantization map. From (4.3) and (4.4) the amplitude for metric boundary data is obtained by expanding the delta functions as

$$
\tilde{\mathcal{A}}_{\mathrm{PR}}\left(X_{i j}\right)=\int\left[\prod_{(i, j) \in \mathcal{N}} \frac{\mathrm{d} g_{i j}}{\kappa^{3}}\right]\left[\frac{\mathrm{d} Y_{j i}}{(2 \pi \hbar \kappa)^{3}}\right]\left[\prod_{l} \mathrm{~d} h_{l}\right]\left[\frac{\mathrm{d} Y_{e}}{(2 \pi \hbar \kappa)^{3}}\right]\left[\prod_{e \notin \partial \Delta} E\left(H_{e^{*}}\left(h_{l}\right), Y_{e}\right)\right]
$$




$$
\times\left[\prod_{\substack{(i, j) \in \mathcal{N} \\ i<j}} E\left(g_{i j} h_{j}^{-1} K_{j i}\left(h_{l}\right) h_{i} g_{j i}^{-1}, Y_{j i}\right)\right]\left[\prod_{(i, j) \in \mathcal{N}} E\left(g_{i j}^{-1}, X_{i j}\right)\right]
$$

We emphasize that here $X_{i j}$ 's are the fixed boundary edge vectors, while $Y_{j i}$ 's are auxiliary boundary edge vectors, which are the Lagrange multipliers imposing the triviality of holonomies around dual faces touching the boundary. We will see that the two are identified (up to orientations and parallel transports) in the classical limit. Importantly, equation (4.5) is nothing else than the simplicial path integral for a complex with boundary, and a fixed discrete metric on this boundary represented by $X_{i j}$ 's. This can be seen by writing the explicit form of the noncommutative plane waves, thus obtaining a formula like (3.4), augmented by boundary terms. We will use this expression in the next section to study the semi-classical limit.

\section{Exact amplitudes for metric boundary data on a sphere}

By integrating over all $Y_{e}$ and using the property (2.5) for the non-commutative plane waves, we may write (4.5) as

$$
\begin{aligned}
& \tilde{\mathcal{A}}_{\mathrm{PR}}\left(X_{i j}\right) \propto \int\left[\prod_{(i, j) \in \mathcal{N}} \frac{\mathrm{d} g_{i j}}{\kappa^{3}}\right]\left[\frac{\mathrm{d} Y_{j i}}{(2 \pi \hbar \kappa)^{3}}\right]\left[\prod_{l} \mathrm{~d} h_{l}\right]\left[\prod_{e \notin \partial \Delta} \delta\left(H_{e^{*}}\left(h_{l}\right)\right)\right] \\
& \times\left[\prod_{\substack{(i, j) \in \mathcal{N} \\
i<j}}\left(E\left(g_{i j}, Y_{j i}\right) E\left(g_{i j}^{-1}, X_{i j}\right)\right) \star E\left(h_{j}^{-1} K_{j i}\left(h_{l}\right) h_{i}, Y_{j i}\right) \star\left(E\left(g_{j i}^{-1}, Y_{j i}\right) E\left(g_{j i}^{-1}, X_{j i}\right)\right)\right],
\end{aligned}
$$

where the $\star$-product acts on $Y_{j i}$. For simplicity, we often do not include explicitly the finite proportionality constants in front of amplitudes, because they are immaterial for our results, and will eventually be cancelled by normalization. Further integrating in (4.6) over all $g_{i j}$ gives

$$
\begin{aligned}
\tilde{\mathcal{A}}_{\mathrm{PR}}\left(X_{i j}\right) \propto \int & {\left[\frac{\mathrm{d} Y_{j i}}{(2 \pi \hbar \kappa)^{3}}\right]\left[\prod_{l} \mathrm{~d} h_{l}\right]\left[\prod_{e \notin \partial \Delta} \delta\left(H_{e^{*}}\left(h_{l}\right)\right)\right] } \\
& \times\left[\prod_{\substack{(i, j) \in \mathcal{N} \\
i<j}} \delta_{\star}\left(Y_{j i}, X_{i j}\right) \star E\left(h_{j}^{-1} K_{j i}\left(h_{l}\right) h_{i}, Y_{j i}\right) \star \delta_{\star}\left(Y_{j i},-X_{j i}\right)\right],
\end{aligned}
$$

where now the $\delta_{\star}$-functions impose the identifications of boundary edge vector variables, up to parallel transport. Indeed, the non-commutative plane wave takes care of the parallel transport between the frames of $X_{i j}$ and $X_{j i}$, as we may easily observe using the property (2.7) of the plane wave as we permute the first $\delta_{\star}$-function with the plane wave to obtain

$$
\begin{aligned}
\tilde{\mathcal{A}}_{\mathrm{PR}}\left(X_{i j}\right) \propto & \int\left[\frac{\mathrm{d} Y_{j i}}{(2 \pi \hbar \kappa)^{3}}\right]\left[\prod_{l} \mathrm{~d} h_{l}\right]\left[\prod_{e \notin \partial \Delta} \delta\left(H_{e^{*}}\left(h_{l}\right)\right)\right] \\
& \times\left[\prod_{\substack{(i, j) \in \mathcal{N} \\
i<j}} E\left(h_{j}^{-1} K_{j i}\left(h_{l}\right) h_{i}, Y_{j i}\right) \star \delta_{\star}\left(\operatorname{Ad}_{h_{j}^{-1} K_{j i}\left(h_{l}\right) h_{i}} Y_{j i}, X_{i j}\right) \star \delta_{\star}\left(Y_{j i},-X_{j i}\right)\right] .
\end{aligned}
$$

We may further integrate over all $Y_{j i}$ to get

$$
\tilde{\mathcal{A}}_{\mathrm{PR}}\left(X_{i j}\right) \propto \int\left[\prod_{l} \mathrm{~d} h_{l}\right]\left[\prod_{e \notin \partial \Delta} \delta\left(H_{e^{*}}\left(h_{l}\right)\right)\right]
$$




$$
\times\left[\prod_{\substack{(i, j) \in \mathcal{N} \\ i<j}} E\left(h_{i}^{-1} K_{i j}\left(h_{l}\right) h_{j}, X_{j i}\right) \star \delta_{\star}\left(\operatorname{Ad}_{h_{j}^{-1} K_{j i}\left(h_{l}\right) h_{i}} X_{j i},-X_{i j}\right)\right] .
$$

We see that the edge vectors $X_{i j}, X_{j i}$ corresponding to the same edge (with opposite orientations) in different frames of reference are identified up to a parallel transport by $h_{j}^{-1} K_{j i}\left(h_{l}\right) h_{i}$ through the non-commutative delta distributions $\delta_{\star}\left(\operatorname{Ad}_{h_{j}^{-1} K_{j i}\left(h_{l}\right) h_{i}} X_{j i},-X_{i j}\right)$.

We wish to further integrate over the variables $h_{i}$. To this aim, we employ the change of variables $X_{j i} \mapsto \operatorname{Ad}_{h_{i}^{-1} K_{i j}\left(h_{l}\right) h_{j}} X_{j i}$, i.e., we parallel transport the variables $X_{j i}$ to the frames of $X_{i j}$ to get a simple identification of the boundary variables, and move all $h_{i}$-dependence to the plane waves. We thus get

$$
\begin{aligned}
\tilde{\mathcal{A}}_{\mathrm{PR}}\left(X_{i j}\right) \propto \int\left[\prod_{l} \mathrm{~d} h_{l}\right]\left[\prod_{e \notin \partial \Delta} \delta\left(H_{e^{*}}\left(h_{l}\right)\right)\right] \\
\times\left[\prod_{\substack{(i, j) \in \mathcal{N} \\
i<j}} E\left(h_{i}^{-1} K_{i j}\left(h_{l}\right) h_{j}, X_{j i}\right)\right] \star\left[\prod_{\substack{(i, j) \in \mathcal{N} \\
i<j}} \delta_{\star}\left(X_{i j},-X_{j i}\right)\right],
\end{aligned}
$$

Note that for every vertex $i$ there is a unique path via the edges $\left(j_{n-1}, j_{n}\right)_{n=1, \ldots, l}$, s.t. $j_{0}=0$, $j_{l}=i$, from the root to the vertex $i$ along the spanning tree. Now, by making the changes of variables

$$
h_{i} \mapsto\left[\coprod_{n=0}^{l} K_{j_{n-1} j_{n}}^{-1}\left(h_{l}\right)\right] h_{i}
$$

where by $\overleftarrow{\prod}$ we denote an ordered product of group elements such that the product index increases from right to left, we obtain

$$
\begin{aligned}
\tilde{\mathcal{A}}_{\mathrm{PR}}\left(X_{i j}\right) \propto & \int\left[\prod_{l} \mathrm{~d} h_{l}\right]\left[\prod_{e \notin \partial \Delta} \delta\left(H_{e^{*}}\left(h_{l}\right)\right)\right]\left[\prod_{\substack{(i, j) \in \operatorname{tree} \\
i<j}} E\left(h_{i}^{-1} h_{j}, X_{j i}\right)\right] \\
& \times\left[\prod_{\substack{(i, j) \notin \operatorname{tree} \\
i<j}} E\left(h_{i}^{-1} L_{i j}\left(h_{l}\right) h_{j}, X_{j i}\right)\right] \star\left[\prod_{\substack{(i, j) \in \mathcal{N} \\
i<j}} \delta_{\star}\left(X_{i j},-X_{j i}\right)\right] \\
= & \int\left[\prod_{l} \mathrm{~d} h_{l}\right]\left[\prod_{e \notin \partial \Delta} \delta\left(H_{e^{*}}\left(h_{l}\right)\right)\right] \\
& \times\left[\prod_{\star}^{\longrightarrow}\left(E\left(h_{i}, \sum_{j} \epsilon_{i j} X_{j i}\right) \star \prod_{j} E\left(L_{i j}\left(h_{l}\right), X_{j i}\right)\right)\right] \star\left[\prod_{\substack{(i, j) \in \mathcal{N} \\
i<j}} \delta_{\star}\left(X_{i j},-X_{j i}\right)\right] .
\end{aligned}
$$

Here, $\epsilon_{i j}:=\operatorname{sgn}(i-j) A_{i j}$, where $A_{i j}$ is the adjacency matrix of the dual graph of the boundary triangulation. Moreover, $L_{i j}\left(h_{l}\right) \equiv G_{i j}^{-1}\left(h_{l}\right) H_{i j}\left(h_{l}\right) G_{i j}\left(h_{l}\right)$, where $H_{i j}\left(h_{l}\right)$ is the product of $K_{k l}\left(h_{l}\right)$ 's around the unique cycle of the boundary dual graph formed by adding the edge $(i, j)$ to the spanning tree, and $G_{i j}\left(h_{l}\right)$ is the product of $K_{k l}\left(h_{l}\right)$ 's along the unique path from the root of the spanning tree to the cycle. The cycles formed from the spanning tree of a graph by adding single edges span the loop space of the graph. Thus, $H_{i j}\left(h_{l}\right)$ are trivial for a trivial boundary topology, if the product of $K_{k l}\left(h_{l}\right)$ 's around all boundary vertices are trivial. On the other hand, the product of $K_{k l}\left(h_{l}\right)$ 's around a boundary vertex is constrained to be trivial by the flatness constraints for the bulk holonomies only if the neighborhood of the vertex is a half-ball, since 
only in this case is the loop around the vertex contractible along the faces of the 2-complex. Thus, given that the neighborhoods of all boundary vertices have trivial topology, the flatness constraints impose $L_{i j}\left(h_{l}\right)$ to be trivial, if the boundary has a trivial topology, i.e., $\partial \Delta \cong S^{2}$. Accordingly, we have

$$
\begin{aligned}
\tilde{\mathcal{A}}_{\mathrm{PR}}\left(X_{i j}\right) \propto \int & {\left[\prod_{l} \mathrm{~d} h_{l}\right]\left[\prod_{e \notin \partial \Delta} \delta\left(H_{e^{*}}\left(h_{l}\right)\right)\right] } \\
& \times\left[\prod_{i}^{\longrightarrow} E\left(h_{i}, \epsilon_{i j} X_{j i}\right)\right] \star\left[\prod_{\substack{i, j) \in \mathcal{N} \\
i<j}} \delta_{\star}\left(X_{i j},-X_{j i}\right)\right],
\end{aligned}
$$

where we used the notation $\overrightarrow{\mathrm{K}}$ for the ordered star product of plane waves. Integrating over $h_{i}$ then yields the closure constraints for the boundary triangles, and we end up with

$$
\tilde{\mathcal{A}}_{\mathrm{PR}}\left(X_{i j}\right) \propto[\delta(0)]^{d}\left[\overrightarrow{\mathrm{W}} \delta_{\star}\left(\sum_{j} \epsilon_{i j} X_{j i}\right)\right] \star\left[\prod_{\substack{(i, j) \in \mathcal{N} \\ i<j}} \delta_{\star}\left(X_{i j},-X_{j i}\right)\right],
$$

where the sum is over vertices $j$ connected to the vertex $i$, and $d$ is the degree of divergence arising from the redundant delta distributions over the dual faces $e^{*} \in \Delta^{*}, e \notin \partial \Delta$.

It is clear that in the abelian limit $\kappa \rightarrow 0$, where the $\star$-product coincides with the pointwise product and $\delta_{\star} \rightarrow \delta$, the above amplitude imposes closure and identification of the edge vectors. However, the case of the classical limit $\hbar \rightarrow 0$ is more subtle: The whole notion of a noncommutative Fourier transform breaks down in this limit, since the non-commutative plane wave becomes ill-defined, having no well-defined limit. We will see in the following the effects of these complications and how to take them into account in studying the classical limit.

\section{Semi-classical analysis for metric boundary data}

In this section we will study the classical limit of the first order phase space path integral (4.5) for the Ponzano-Regge model obtained through the non-commutative Fourier transform. In particular, we will study the classical limit via the stationary phase approximation, first by using the usual 'commutative' variational method. However, we discover that the resulting classical geometricity constraints on the classical metric variables depend on the initial choice of quantization map - a rather problematic outcome. Therefore, we are compelled to adopt the noncommutative variational method for the stationary phase approximation in order to obtain the correct classical equations of motion, as in the analogous case of quantum mechanics of a point particle on $\mathrm{SO}(3)$, considered previously in [45]. We will again see that the non-commutative method leads to the correct and unambiguous classical geometricity constraints on the simplicial metric variables, and offer some further justification for the use of the non-commutative variational calculus. Moreover, the analysis shows how subtle the notion of "classical limit" is for the Ponzano-Regge amplitudes, which are in the end convolutions of non-commutative planes waves, in the flux representation. We would expect similar subtleties to be relevant for $4 \mathrm{~d}$ gravity models as well.

Let us begin by bringing the path integral (4.5) into a form suitable for stationary phase approximation via variational calculus. We may use the expression (2.3) for the non-commutative plane wave to express (4.5) as

$$
\tilde{\mathcal{A}}_{\mathrm{PR}}\left(X_{i j}\right)=\int\left[\prod_{(i, j) \in \mathcal{N}} \frac{\mathrm{d} g_{i j}}{\kappa^{3}}\right]\left[\prod_{\substack{(i, j) \in \mathcal{N} \\ i<j}} \frac{\mathrm{d} Y_{i j}}{(2 \pi \hbar \kappa)^{3}}\right]\left[\prod_{l} \mathrm{~d} h_{l}\right]\left[\prod_{e \notin \partial \Delta} \frac{\mathrm{d} Y_{e}}{(2 \pi \hbar \kappa)^{3}}\right]
$$




$$
\begin{aligned}
& \times\left[\prod_{e \notin \partial \Delta} c\left(H_{e^{*}}\left(h_{l}\right)\right) e^{\frac{i}{\hbar} Y_{e} \cdot \zeta\left(H_{e^{*}}\left(h_{l}\right)\right)}\right]\left[\prod_{(i, j) \in \mathcal{N}} c\left(g_{i j}^{-1}\right) e^{\frac{i}{\hbar} X_{i j} \cdot \zeta\left(g_{i j}^{-1}\right)}\right] \\
& \times\left[\prod_{\substack{(i, j) \in \mathcal{N} \\
i<j}} c\left(g_{i j} h_{j}^{-1} K_{j i}\left(h_{l}\right) h_{i} g_{j i}^{-1}\right) e^{\frac{i}{\hbar} Y_{i j} \cdot \zeta\left(g_{i j} h_{j}^{-1} K_{j i}\left(h_{l}\right) h_{i} g_{j i}^{-1}\right)}\right],
\end{aligned}
$$

and by further combining the exponentials we obtain

$$
\begin{aligned}
& \tilde{\mathcal{A}}_{\mathrm{PR}}\left(X_{i j}\right)=\int\left[\prod_{\substack{(i, j) \in \mathcal{N} \\
\kappa^{3}}} \frac{\mathrm{d} g_{i j}}{\kappa^{3}}\left(g_{i j}^{-1}\right)\right]\left[\prod_{\substack{(i, j) \in \mathcal{N} \\
i<j}} \frac{\mathrm{d} Y_{i j}}{(2 \pi \hbar \kappa)^{3}}\right]\left[\prod_{l} \mathrm{~d} h_{l}\right]\left[\prod_{e \notin \partial \Delta} \frac{\mathrm{d} Y_{e}}{(2 \pi \hbar \kappa)^{3}}\right] \\
& \times\left[\prod_{e \notin \partial \Delta} c\left(H_{e^{*}}\left(h_{l}\right)\right)\right]\left[\prod_{\substack{(i, j) \in \mathcal{N} \\
i<j}} c\left(g_{i j} h_{j}^{-1} K_{j i}\left(h_{l}\right) h_{i} g_{j i}^{-1}\right)\right] \\
& \times \exp \left\{\frac{i}{\hbar}\left[\sum_{e \notin \partial \Delta} Y_{e} \cdot \zeta\left(H_{e^{*}}\left(h_{l}\right)\right)+\sum_{\substack{(i, j) \in \mathcal{N} \\
i<j}} Y_{i j} \cdot \zeta\left(g_{i j} h_{j}^{-1} K_{j i}\left(h_{l}\right) h_{i} g_{j i}^{-1}\right)+\sum_{(i, j) \in \mathcal{N}} X_{i j} \cdot \zeta\left(g_{i j}^{-1}\right)\right]\right\} .
\end{aligned}
$$

In this form the amplitude is amenable to a stationary phase analysis through the study of the extrema of the exponential

$$
\mathcal{S}_{\mathrm{PR}}:=\sum_{e \notin \partial \Delta} Y_{e} \cdot \zeta\left(H_{e^{*}}\left(h_{l}\right)\right)+\sum_{\substack{(i, j) \in \mathcal{N} \\ i<j}} Y_{i j} \cdot \zeta\left(g_{i j} h_{j}^{-1} K_{j i}\left(h_{l}\right) h_{i} g_{j i}^{-1}\right)+\sum_{(i, j) \in \mathcal{N}} X_{i j} \cdot \zeta\left(g_{i j}^{-1}\right) .
$$

We stress that this is just the classical action of discretized BF theory in its first order variables, the edge vectors $Y_{e}$ and the parallel transports $h_{l}$, augmented by boundary terms. Therefore, we expect to obtain in the classical limit the classical BF 'equations of motion', that is, geometricity constraints imposing flatness of holonomies around dual faces and closure of edge vectors for all triangles (up to the appropriate parallel transports).

\subsection{Stationary phase approximation via commutative variational method}

We first proceed to consider the usual 'commutative' stationary phase approximation of the first order Ponzano-Regge path integral (4.5) by studying the extrema of the action (5.2). There are five different kinds of integration variables in (4.5): $Y_{e}$ for $e \notin \partial \Delta, Y_{i j}, h_{l}$ in the bulk, $h_{i}$ touching the boundary and $g_{i j}$, whose variations we will consider in the following.

Variation of $Y_{e}$ : Requiring the variation of the action with respect to $Y_{e}$ to vanish simply gives

$$
\zeta\left(H_{e^{*}}\left(h_{l}\right)\right)=0 \Leftrightarrow H_{e^{*}}\left(h_{l}\right)=\mathbb{1}
$$

for all $e \notin \partial \Delta$, i.e., the flatness of the connection around the dual faces $e^{*}$ in the bulk.

Variation of $Y_{i j}$ : Similarly, variation with respect to $Y_{i j}$ gives

$$
\zeta\left(g_{i j} h_{j}^{-1} K_{j i}\left(h_{l}\right) h_{i} g_{j i}^{-1}\right)=0 \Leftrightarrow g_{i j} h_{j}^{-1} K_{j i}\left(h_{l}\right) h_{i} g_{j i}^{-1}=\mathbb{1}
$$

for all $(i, j) \in \mathcal{N}, i<j$, i.e., the triviality of the connection around the faces $e^{*}$ dual to boundary edges $e \in \partial \Delta$.

Variation of $h_{l}$ in the bulk: The variations for the group elements are slightly less trivial. Taking left-invariant Lie derivatives of the exponential with respect to a group element $h_{l^{\prime}} \equiv g_{t f}$ in the bulk, we obtain

$$
\sum_{e \notin \partial \Delta} Y_{e} \cdot \mathcal{L}_{k}^{h_{l^{\prime}}} \zeta\left(H_{e^{*}}\left(h_{l}\right)\right)+\sum_{\substack{(i, j) \in \mathcal{N} \\ i<j}} Y_{j i} \cdot \mathcal{L}_{k}^{h_{l^{\prime}}} \zeta\left(g_{i j} h_{j}^{-1} K_{j i}\left(h_{l}\right) h_{i} g_{j i}^{-1}\right)=0 \quad \forall k .
$$


Here, only the three terms in the sums depending on the holonomies around the boundaries of the three dual faces, which contain $l^{\prime}:=t f$ are non-zero. (Each dual edge $f^{*}$ belongs to exactly three dual faces $e^{*}$ of $\Delta^{*}$, since $\Delta^{*}$ is dual to a 3-dimensional triangulation.) Now, using the fact uncovered through the previous variations that the holonomies around the dual faces are trivial for the stationary phase configurations, and the property $\zeta\left(\operatorname{ad}_{g} h\right)=$ $\operatorname{Ad}_{g} \zeta(h)$ of the coordinates, we obtain

$$
\sum_{\substack{e \in \Delta \\ e^{*} \ni f^{*}}} \epsilon_{f e}\left(\operatorname{Ad}_{G_{f e}} Y_{e}\right)=0,
$$

where $\operatorname{Ad}_{G_{f e}}$ implements the parallel transport from the frame of $Y_{e}$ to the frame of $f$, and $\epsilon_{f e}= \pm 1$ accounts for the orientation of $h_{l}$ with respect to the holonomy $H_{e^{*}}\left(h_{l}\right)$ and thus the relative orientations of the edge vectors. Clearly, this imposes the metric closure constraint for the three edge vectors of each bulk triangle $f \notin \partial \Delta$ in the frame of $f$. This same condition gives the metric compatibility of the discrete connection, which in turn, if substituted back in the classical action, before considering the other saddle point equations, turn the discrete 1st order action into the 2nd order action for the triangulation $\Delta$.

Variation of $h_{i}$ : Varying a $h_{i}$ we get

$$
\begin{aligned}
& \sum_{\substack{(i, j) \in \mathcal{N} \\
i<j}} Y_{i j} \cdot \mathcal{L}_{k}^{h_{i}} \zeta\left(g_{i j} h_{j}^{-1} K_{j i}\left(h_{l}\right) h_{i} g_{j i}^{-1}\right) \\
& \quad+\sum_{\substack{(j, i) \in \mathcal{N} \\
j<i}} Y_{j i} \cdot \mathcal{L}_{k}^{h_{i}} \zeta\left(g_{j i} h_{i}^{-1} K_{i j}\left(h_{l}\right) h_{j} g_{i j}^{-1}\right)=0 \quad \forall k
\end{aligned}
$$

Again there are three non-zero terms in this expression, which correspond to the boundary triangles $f_{j} \in \partial \Delta$ neighboring $f_{i}$, i.e., such that $(i, j) \in \mathcal{N}$. We obtain the closure of the boundary integration variables $Y_{i j}$ as

$$
\sum_{\substack{f_{j} \in \partial \Delta \\(i, j) \in \mathcal{N}}} \epsilon_{j i}\left(\operatorname{Ad}_{g_{j i}}^{-1} Y_{i j}\right)=0,
$$

where $\operatorname{Ad}_{g_{j i}}^{-1}$ parallel transports the edge vectors $Y_{j i}$ to the frame of the boundary triangle $f_{i}$, and $\epsilon_{j i}= \pm 1$ again accounts for the relative orientation.

Variation of $g_{i j}$ : Taking Lie derivatives of the exponential with respect to a $g_{i j}$, we obtain

$$
\begin{aligned}
& Y_{i j} \cdot \mathcal{L}_{k}^{g_{i j}} \zeta\left(g_{i j} h_{j}^{-1} K_{j i}\left(h_{l}\right) h_{i} g_{j i}^{-1}\right)+X_{i j} \cdot \mathcal{L}_{k}^{g_{i j}} \zeta\left(g_{i j}^{-1}\right)=0 \quad \forall k \\
& \Leftrightarrow \quad \operatorname{Ad}_{g_{i j}}^{-1} Y_{i j}-D^{\zeta}\left(g_{i j}\right) X_{i j}=0=\operatorname{Ad}_{g_{i j}}^{-1} Y_{i j}+D^{\zeta}\left(g_{j i}\right) X_{j i}
\end{aligned}
$$

for all $i<j$, where we denote $\left(D^{\zeta}(g)\right)_{k l}:=\tilde{\mathcal{L}}_{k} \zeta_{l}(g)$. We see that this equation identifies the boundary metric variables $X_{i j}$ with the integration variables $Y_{i j}$, taking into account the orientation and the parallel transport between the frames of each vector, plus a nongeometric deformation given by the matrix $D^{\zeta}\left(g_{i j}\right) .{ }^{6}$

Thus, we have obtained the constraint equations corresponding to variations of all the integration variables. In particular, by combining the equations (5.4) with the boundary closure constraint (5.3), we obtain

$$
\sum_{\substack{f_{j} \in \partial \Delta \\(i, j) \in \mathcal{N}}} D^{\zeta}\left(g_{i j}\right) X_{i j}=0 \quad \forall i,
$$

\footnotetext{
${ }^{6}$ Also, in varying $g_{i j}$ we must assume that the measure $c(g) \mathrm{d} g$ on the group is continuous, which should be true for any reasonable choice of a quantization map, as it indeed is for all the cases we consider below.
} 
which gives, in general, a deformed closure constraint for the boundary metric edge variables $X_{i j}$. In addition, from (5.4) alone we obtain a deformed identification

$$
\operatorname{Ad}_{g_{i j}}\left(D^{\zeta}\left(g_{i j}\right) X_{i j}\right)=-\operatorname{Ad}_{g_{j i}}\left(D^{\zeta}\left(g_{j i}\right) X_{j i}\right),
$$

naturally up to a parallel transport, of the boundary edge variables $X_{i j}$ and $X_{j i}$. Accordingly, we obtain for the amplitude

$$
\begin{aligned}
\tilde{\mathcal{A}}_{\mathrm{PR}}\left(X_{i j}\right) \propto & \int\left[\prod_{\substack{(i, j) \in \mathcal{N} \\
\kappa^{3}}} \frac{\mathrm{d} g_{i j}}{\kappa^{3}}\left(g_{i j}^{-1}\right)\right]\left[\prod_{v \in \partial \Delta} \delta\left(H_{v}\left(g_{i j}\right)\right)\right]\left[\prod_{f_{i} \in \partial \Delta} \delta_{\star}\left(\sum_{\substack{f_{j} \in \partial \Delta \\
(i, j) \in \mathcal{N}}} D^{\zeta}\left(g_{i j}\right) X_{i j}\right)\right] \\
& \star\left[\prod_{\substack{(i, j) \in \mathcal{N} \\
i<j}} \delta_{\star}\left(\operatorname{Ad}_{g_{i j}}\left(D^{\zeta}\left(g_{i j}\right) X_{i j}\right)+\operatorname{Ad}_{g_{j i}}\left(D^{\zeta}\left(g_{j i}\right) X_{j i}\right)\right)\right] \\
& \star \exp \left\{\frac{i}{\hbar} \sum_{(i, j) \in \mathcal{N}} X_{i j} \cdot \zeta\left(g_{i j}^{-1}\right)\right\}(1+\mathcal{O}(\hbar)) .
\end{aligned}
$$

The proportionality constant is given by the configuration space volume for the geometric configurations in the bulk, which is generically infinite but is cancelled by normalization. The delta functions impose the constraints on boundary data discussed above. In particular, $H_{v}\left(g_{i j}\right)$ are the holonomies around the boundary vertices $v \in \partial \Delta$, whose triviality follows from the triviality of the bulk holonomies. Notice that one must write the integrand in terms of $\star$-products and $\star$-delta functions in order for the constraints to be correctly imposed, since the amplitude acts on wave functions through $\star$-multiplication. The exact form of the deformation matrix $D_{k l}^{\zeta}(g) \equiv\left\{X_{k}, \zeta_{l}\right\}(g)=\delta_{k l}+\mathcal{O}(\kappa,|\ln (g)|)$, and accordingly the geometric content of these constraints, depends on the coordinates $\zeta$, which are determined by the discretization of the continuum BF action or, equivalently, the initial choice of the quantization map. We see that only in the abelian limit $\kappa \rightarrow 0,|\zeta|=$ const, do the different choices agree in general, producing the undeformed discrete geometric constraints

$$
\sum_{\substack{f_{j} \in \partial \Delta \\(i, j) \in \mathcal{N}}} X_{i j}=0 \quad \forall f_{i} \in \partial \Delta \quad \text { and } \quad \operatorname{Ad}_{g_{i j}} X_{i j}=\operatorname{Ad}_{g_{j i}} X_{j i} \quad \forall(i, j) \in \mathcal{N}
$$

for the discretized boundary metric variables $X_{i j} \in \mathfrak{s u}(2)^{*}$.

\section{Some examples}

Before we go on to consider the stationary phase boundary configurations obtained through the ordinary commutative variational calculus for some specific choices of the coordinates $\zeta$, and thus the associated quantization maps, let us make a few general remarks on the apparent dependence of the limit on this choice. As we have already emphasized above, the exact functional form of the non-commutative plane waves, and thus the coordinate choice, is determined ultimately by the choice of the quantization map and the $\star$-product that we thus obtain. We have found the general expression for the plane wave as a $\star$-exponential

$$
E(g, X)=e_{\star}^{\frac{i}{\hbar \kappa} k(g) \cdot X}=\sum_{n=0}^{\infty} \frac{1}{n !}\left(\frac{i}{\hbar \kappa}\right)^{n} k^{i_{1}}(g) \cdots k^{i_{n}}(g) X_{i_{1}} \star \cdots \star X_{i_{n}} .
$$

From this expression we may observe that the way the Planck constant $\hbar$ enters into the plane wave is very subtle. There are negative powers of $(\hbar \kappa)$ coming from the prefactor in the exponential, while from the $\star$-monomials arise positive powers of $(\hbar \kappa)$. The way these different 
contributions go together determines the explicit functional form of the non-commutative plane wave, and accordingly the behavior in the classical limit $\hbar \rightarrow 0$. Therefore, it is not too surprising that we may eventually find different classical limits for different choices of plane waves through the application of the ordinary stationary phase method. In particular, it is important to realize that the non-commutative plane wave itself is purely a quantum object with an ill-defined classical limit, and therefore has no duty to coincide with anything in this limit. For this reason, the stationary phase solutions corresponding to different $\star$-products may also differ from each other, even though the $\star$-product itself coincides with the pointwise product in this limit. On the contrary, in the abelian limit $\kappa \rightarrow 0$ we also scale the coordinates $k^{i}$ on the group, so that $k^{i} / \kappa$ stay constant, since $\kappa$ determines the scale associated to the group manifold. Therefore, the non-commutative plane wave agrees with the usual commutative plane wave in this limit. Only in the abelian limit may one expect the different choices of non-commutative structures lead to unambiguous results, when one applies the commutative variational calculus.

Symmetric \& Duflo quantization maps. The symmetric quantization map $\mathfrak{Q}_{\mathrm{S}}: \operatorname{Pol}\left(\mathfrak{s u}(2)^{*}\right) \rightarrow$ $U(\mathfrak{s u}(2))$ is determined by the symmetric operator ordering for monomials

$$
\mathfrak{Q}_{\mathrm{S}}\left(X_{i_{1}} X_{i_{2}} \cdots X_{i_{n}}\right) \stackrel{!}{=} \frac{1}{n !} \sum_{\sigma \in \Sigma_{n}} \hat{X}_{i_{\sigma(1)}} \hat{X}_{i_{\sigma(2)}} \cdots \hat{X}_{i_{\sigma(n)}}
$$

where $\Sigma_{n}$ is the group of permutations of $n$ elements, and extends by linearity to any completion of the polynomial algebra $\operatorname{Pol}\left(\mathfrak{s u}(2)^{*}\right)$. In particular, we have

$$
\mathfrak{Q}_{\mathrm{S}}^{-1}\left(e^{\frac{i}{\hbar \kappa} k(g) \cdot \hat{X}}\right)=\sum_{n=0}^{\infty} \frac{i^{n}}{n !(\hbar \kappa)^{n}} k^{i_{1}}(g) \cdots k^{i_{n}}(g) \mathfrak{Q}_{\mathrm{S}}^{-1}\left(\hat{X}_{i_{1}} \cdots \hat{X}_{i_{n}}\right)=e^{\frac{i}{\hbar \kappa} k(g) \cdot X} \equiv E_{\mathrm{S}}(g, X)
$$

and accordingly to this quantization prescription is associated a non-commutative plane wave with $c_{\mathrm{S}}(g)=1, \zeta_{\mathrm{S}}(g)=-\frac{i}{\kappa} \ln (g) \in \mathfrak{s u}(2)$, where the value of the logarithm is taken in the principal branch [27].

The Duflo quantization map $\mathfrak{Q}_{\mathrm{D}}$ is defined as

$$
\mathfrak{Q}_{\mathrm{D}}=\mathfrak{Q}_{\mathrm{S}} \circ j^{\frac{1}{2}}\left(\vec{\partial}_{x}\right),
$$

where $j^{\frac{1}{2}}\left(\vec{\partial}_{x}\right)$ is a differential operator associated to the function $j: \mathfrak{s u}(2) \rightarrow \mathbb{C}$ given by

$$
j^{\frac{1}{2}}(X):=\operatorname{det}\left(\frac{\sinh \left(\frac{1}{2} \operatorname{ad}_{X}\right)}{\frac{1}{2} \operatorname{ad}_{X}}\right)^{\frac{1}{2}}
$$

The definition is such that $\mathfrak{Q}_{\mathrm{D}}$ restricts to an isomorphism from the $g$-invariant functions on $\mathfrak{s u}(2)^{*}$ to $g$-invariant operators (i.e., Casimirs) in $\overline{U(\mathfrak{s u}(2))}$, and therefore the Duflo map can be considered as algebraically the most natural choice for a quantization map. In the Duflo case we obtain $c_{\mathrm{D}}(g)=\kappa\left|\zeta_{\mathrm{S}}(g)\right| / \sin \left(\kappa\left|\zeta_{\mathrm{S}}(g)\right|\right)$, but the coordinates are the same $\zeta_{\mathrm{D}} \equiv \zeta_{\mathrm{S}}$ as for the symmetric quantization map, so the amplitudes have the same stationary phase behavior in both cases. In this respect it is important to note that even though the Duflo factor $c_{\mathrm{D}}(g)=$ $\kappa\left|\zeta_{\mathrm{S}}(g)\right| / \sin \left(\kappa\left|\zeta_{\mathrm{S}}(g)\right|\right)$ diverges for $\kappa\left|\zeta_{\mathrm{S}}(g)\right|=\pi$, the path integral measure is still well-behaved, since $c_{\mathrm{D}}(g) \mathrm{d} g=\left(\sin \left(\kappa\left|\zeta_{\mathrm{S}}\right|\right) / \kappa\left|\zeta_{\mathrm{S}}\right|\right) \mathrm{d}^{3} \zeta_{\mathrm{S}}$ remains finite.

We obtain from the equation (A.1) in Appendix A for the deformation matrix as a function of the coordinates

$$
D_{k l}^{\mathrm{S}}\left(\zeta_{\mathrm{s}}\right)=\left(\frac{\kappa\left|\zeta_{\mathrm{s}}\right|}{\sin \left(\kappa\left|\zeta_{\mathrm{s}}\right|\right)}\right)\left[\cos \left(\kappa\left|\zeta_{\mathrm{s}}\right|\right) \delta_{k l}+\left(\frac{\sin \left(\kappa\left|\zeta_{\mathrm{s}}\right|\right)}{\kappa\left|\zeta_{\mathrm{s}}\right|}-\cos \left(\kappa\left|\zeta_{\mathrm{s}}\right|\right)\right) \frac{\zeta_{\mathrm{s}, k} \zeta_{\mathrm{s}, l}}{\left|\zeta_{\mathrm{s}}\right|^{2}}-\kappa \epsilon_{k l m} \zeta_{\mathrm{s}}^{m}\right]
$$


This deformation matrix has the following nice property: $D_{k l}^{\mathrm{S}}(k) k^{l}=k_{k}$. This implies, in particular, that when the edge vectors are stable under the dual connection variables, $\operatorname{Ad}_{g_{i j}} X_{i j}=X_{i j}$ $\Leftrightarrow k\left(g_{i j}\right) \propto X_{i j}$, we have $D^{\mathrm{S}}\left(g_{i j}\right) X_{i j}=X_{i j}$, and therefore recover the undeformed closure constraints from (5.5). Accordingly, classical geometric boundary data with $\operatorname{Ad}_{g_{i j}} X_{i j}=X_{i j}$, $X_{i j}=-X_{j i}$ and $\sum_{j} X_{i j}=0$ satisfies the constraint equations for the symmetric quantization map. Except for the stability ansatz $\operatorname{Ad}_{g_{i j}} X_{i j}=X_{i j}$, however, there are undoubtedly other solutions to the constraint equations that do not satisfy this stability requirement, but we have not explored the possibilities in this general case. It is nevertheless clear that these additional solutions do not correspond to simplicial geometries, since for them the closure constraint is again deformed.

Freidel-Livine-Majid quantization map. We will then consider the popular choice of FreidelLivine-Majid quantization map [4, 5, 6, 7, 8, 24, 25, 35], which can be expressed in terms of the symmetric quantization map $\mathfrak{Q}_{\mathrm{S}}$ and a change of parametrization $\chi: \mathfrak{s u}(2) \rightarrow \mathfrak{s u}(2)$ on $\mathrm{SU}(2)$ as $[27]$

$$
\mathfrak{Q}_{\mathrm{FLM}}=\mathfrak{Q}_{\mathrm{S}} \circ \chi
$$

where $\chi(k)=\frac{\sin ^{-1}|k|}{|k|} k$. The inverse coordinate transformation $\chi^{-1}(k)=\frac{\sin |k|}{|k|} k$, however, is two-to-one: it identifies the coordinates of the antipodes $g$ and $-g$ as

$$
\chi^{-1}(k(g))=\chi^{-1}\left(k(g)-\frac{\pi}{2} \frac{k(g)}{|k(g)|}\right)=\chi^{-1}(k(-g)) \quad \forall g \in \mathrm{SU}(2) \backslash\{e\} .
$$

Therefore, the coordinates $\chi^{-1}(k)$ only cover the upper hemisphere $\mathrm{SU}(2) / \mathbb{Z}_{2} \cong \mathrm{SO}(3)$, and the resulting non-commutative Fourier transform is applicable only to functions on $\mathrm{SO}(3)$.

The FLM quantization map yields

$$
\mathcal{Q}_{\mathrm{FLM}}^{-1}\left(e^{\frac{i}{\hbar \kappa} k(g) \cdot \hat{X}}\right)=e^{\frac{i}{\hbar \kappa} \frac{\sin |k(g)|}{|k(g)|} k(g) \cdot X} \equiv E_{\mathrm{FLM}}(g, X) .
$$

Accordingly, it leads to a form of the non-commutative plane wave with $c_{\mathrm{FLM}}(g)=1, \zeta_{\mathrm{FLM}}(g)=$ $\frac{1}{\kappa} \frac{\sin |k(g)|}{|k(g)|} k(g)=-\frac{i}{2 \kappa} \operatorname{tr}_{\frac{1}{2}}\left(g \sigma^{k}\right) \sigma_{k} \in \mathfrak{s u}(2)$, where $\operatorname{tr}_{\frac{1}{2}}$ denotes the trace in the fundamental spin- $\frac{1}{2}$ representation of $\mathrm{SU}(2)$. Due to the linearity of the trace, it is straightforward to calculate the deformation matrix

$$
D_{k l}^{\mathrm{FLM}}(g)=\frac{1}{2} \operatorname{tr}_{\frac{1}{2}}(g) \delta_{k l}+\frac{i}{2} \operatorname{tr}_{\frac{1}{2}}\left(g \sigma^{j}\right) \epsilon_{j k l} \equiv \sqrt{1-\kappa^{2}\left|\zeta_{\mathrm{FLM}}(g)\right|^{2}} \delta_{k l}-\kappa \zeta_{\mathrm{FLM}}^{j}(g) \epsilon_{j k l} .
$$

Thus, according to our general description above, the classical discrete geometricity constraints are satisfied by the deformed boundary metric variables

$$
D^{\mathrm{FLM}}\left(g_{i j}\right) X_{i j}=\sqrt{1-\kappa^{2}\left|\zeta_{\mathrm{FLM}}\left(g_{i j}\right)\right|^{2}} X_{i j}-\kappa\left(\zeta_{\mathrm{FLM}}\left(g_{i j}\right) \wedge X_{i j}\right) .
$$

We have not solved these constraints explicitly, which would generically impose relations between the stationary phase boundary connection $g_{i j}$ and the given boundary metric data $X_{i j}$. However, one can easily confirm that data corresponding to generic classical discrete geometries does not satisfy the constraints, and therefore the geometry resulting from the constraints does not, in general, describe discrete geometries. In fact, the deformed and the undeformed closure constraints are compatible only for $g_{i j} \equiv \mathbb{1}$, or equivalently, in the abelian limit. Therefore, we conclude that the non-commutative metric boundary variables do not have a classical geometric interpretation in the case of FLM quantization map outside the abelian approximation, when one studies the commutative variation of the action. 


\subsection{Stationary phase approximation via non-commutative variational method}

We emphasize that in the above variation of the amplitude we did not take into account the deformation of phase space structure, which appeared crucial for obtaining the correct classical equations of motion in [45] in the case of quantum mechanics of a point particle on $\mathrm{SO}(3)$. This could be guessed to be the origin of the discrepancies between the amplitudes corresponding to different choices of quantization maps in the semi-classical limit. Indeed, we may define the non-commutative variation $\delta_{\star} S$ of the action $S$ in the amplitude via

$$
e_{\star}^{i \delta_{\star} S+\mathcal{O}\left(\delta^{2}\right)} \equiv e_{\star}^{-i S} \star e_{\star}^{i S^{\delta}},
$$

where the $\star$-product acts on the fixed boundary variables $X_{i j}, \mathcal{O}\left(\delta^{2}\right)$ refers to terms higher than first order in the variations, and $S^{\delta} \equiv S\left(g_{i j} \delta g_{i j}, X_{i j}+\delta X_{i j}\right)$ is the varied action. It is easy to see that the non-commutative variation so defined undeforms the above identification (5.4) of $X_{i j}$ and $Y_{i j}$ (up to orientation and parallel transport), simply because we have

$$
E\left(g^{-1}, X\right) \star E\left(g e^{i \epsilon Z}, X\right)=\underbrace{E\left(g^{-1}, X\right) \star E(g, X)}_{=1} \star E\left(e^{i \epsilon Z}, X\right)=e^{\frac{i}{\hbar \kappa} \epsilon(Z \cdot X)+\mathcal{O}\left(\epsilon^{2}\right)}
$$

for any $Z \in \mathfrak{s u}(2)$ and $\epsilon \in \mathbb{R}$ implementing the variation of $g$, so that $\delta g=e^{i \epsilon Z}$. Then, all the above results for variations remain the same by requiring the non-commutative variation $\delta_{\star} S$ of the action to vanish except for equation (5.4), which becomes undeformed, i.e., we obtain the geometric identification $\operatorname{Ad}_{g_{i j}} X_{i j}=Y_{i j}=-\operatorname{Ad}_{g_{j i}} X_{j i}$. Thus, the non-geometric deformation of the constraints does not appear, and we recover exactly the simplicial geometry relations for the boundary metric variables, regardless of the choice of a quantization map.

The non-commutative geometric interpretation of the leading order contribution to the amplitude obtained through the non-commutative variation is largely an open question at the moment - and a very interesting one as well. Clearly, the non-commutative leading order is different from the ordinary commutative result, because we are not considering the usual commutative limit, but another kind of limit that takes into account the non-commutative structure of the phase space. Indeed, this difference is more than welcome, because the commutative result depends on the choice of a quantization map, which is unacceptable, as we have emphasized. Our calculations below show, in fact, that the application of the commutative variational method to an integral kernel that is a function of non-commutative variables leads to a result that does not represent the leading order in $\hbar$ : We confirm in Section 6 that the results obtained (only) by the non-commutative stationary phase analysis agree with those obtained through the indisputable commutative analysis in the coherent state representation. We still lack a complete understanding of the non-commutative variations, but we suspect that the need for the noncommutative variational method arises, because the amplitude $\tilde{\mathcal{A}}_{\mathrm{PR}}\left(X_{i j}\right)$ acts as the integral kernel for the propagator with respect to the $\star$-product and not the pointwise product. As the $\star$-product itself exhibits $\hbar$-dependence, this may modify the asymptotic behavior. The classical constraint equations that we recover via the non-commutative variations are presumably the ones that are imposed on the boundary states by the propagator (again, acting with the $\star$-product) in the classical limit. However, this needs to be substantiated by further research.

To begin with, let us consider the partially 'off-shell' amplitude, where we only substitute the identifications $\operatorname{Ad}_{g_{i j}} X_{i j}=Y_{i j}=-\operatorname{Ad}_{g_{j i}} X_{j i}$ arising from the variations of the boundary connection $g_{i j}$. The substitution is done, again, by multiplying the amplitude by $\star$-delta functions imposing the identities, and integrating over $Y_{i j}$. We also integrate over $X_{j i}$ for $i<j$ in order to explicitly impose the identifications $\operatorname{Ad}_{g_{i j}} X_{i j}=-\operatorname{Ad}_{g_{j i}} X_{j i}$ on the boundary variables. Using the properties of the non-commutative plane waves, and denoting by $\tilde{\mathcal{A}}_{\mathrm{PR}}^{\star l o}\left(X_{i j}\right)$ the leading order 
contribution in $\hbar$ to the amplitude obtained via the non-commutative stationary phase method, we find from (5.1) through a simple substitution

$$
\begin{aligned}
\tilde{\mathcal{A}}_{\mathrm{PR}}^{\star \mathrm{lo}}\left(X_{i j}\right) \propto & \int\left[\prod_{\substack{i, j) \in \mathcal{N} \\
i<j}} \frac{\mathrm{d} g_{i j}}{\kappa^{3}} c\left(g_{i j}^{-1}\right) c\left(g_{i j}^{-1} g_{j i} g_{i j}\right)\right]\left[\prod_{l} \mathrm{~d} h_{l}\right]\left[\prod_{e \notin \partial \Delta} \frac{\mathrm{d} Y_{e}}{(2 \pi \hbar \kappa)^{3}}\right]\left[\prod_{e \notin \partial \Delta} c\left(H_{e^{*}}\left(h_{l}\right)\right)\right] \\
& \times\left[\prod_{\substack{(i, j) \in \mathcal{N} \\
i<j}} c\left(h_{j}^{-1} K_{j i}\left(h_{l}\right) h_{i} g_{j i}^{-1} g_{i j}\right)\right] \exp \left\{\frac{i}{\hbar} \sum_{e \notin \partial \Delta} Y_{e} \cdot \zeta\left(H_{e^{*}}\left(h_{l}\right)\right)\right\} \\
& \times \exp \left\{\sum_{\substack{(i, j) \in \mathcal{N} \\
i<j}} X_{i j} \cdot \zeta\left(h_{j}^{-1} K_{j i}\left(h_{l}\right) h_{i} g_{j i}^{-1} g_{i j}\right)\right\} \\
& \star \exp \left\{\sum_{\substack{i, j) \in \mathcal{N} \\
i<j}} X_{i j} \cdot \zeta\left(g_{i j}^{-1} g_{j i} g_{i j}\right)\right\} \star \exp \left\{\sum_{\substack{i, j) \in \mathcal{N} \\
i<j}} X_{i j} \cdot \zeta\left(g_{i j}^{-1}\right)\right\} \\
= & \left.\int \prod_{l} \mathrm{~d} h_{l}\right]\left[\prod_{e \notin \partial \Delta} \mathrm{d} Y_{e}\right]\left[\prod_{\substack{(i, j) \in \mathcal{N} \\
i<j}} c\left(h_{j}^{-1} K_{j i}\left(h_{l}\right) h_{i}\right)\right]\left[\prod_{e \notin \partial \Delta} c\left(H_{e^{*}}\left(h_{l}\right)\right)\right] \\
& \times \exp \left\{\frac{i}{\hbar}\left[\sum_{e \notin \partial \Delta} Y_{e} \cdot \zeta\left(H_{e^{*}}\left(h_{l}\right)\right)+\sum_{\substack{(i, j) \in \mathcal{N} \\
i<j}} X_{i j} \cdot \zeta\left(h_{j}^{-1} K_{j i}\left(h_{l}\right) h_{i}\right)\right]\right\} .
\end{aligned}
$$

In fact, there is a subtlety in this calculation in choosing the correct ordering of the noncommutative plane waves, which depend on the same non-commutative edge vector after integrating over the $\star$-delta functions, in the first expression of (5.8). We were guided here in the choice by the appropriate geometric form of the result. Indeed, the exponential clearly reflects the typical structure of a $3 \mathrm{~d}$ discrete gravity action: (i) It contains bulk terms $Y_{e} \cdot \zeta\left(H_{e^{*}}\left(h_{l}\right)\right)$, which couple bulk edge vectors and the holonomies around the dual faces, thus associated with deficit angles. (ii) It has boundary terms $X_{i j} \cdot \zeta\left(h_{j}^{-1} K_{j i}\left(h_{l}\right) h_{i}\right)$, which couple boundary edge vectors with the group elements that represent parallel transports between adjacent boundary triangles to the edge, thus associated with dihedral angles.

To make the connection to Regge calculus even clearer, let us adopt the non-commutative structure arising from the symmetric quantization map, and set $H_{e^{*}}\left(h_{l}\right) \equiv \exp \left(i \theta_{e} \hat{n}_{e} \cdot \vec{\sigma}\right)$ and $h_{j}^{-1} K_{j i}\left(h_{l}\right) h_{i} \equiv \exp \left(i \theta_{j i} \hat{n}_{j i} \cdot \vec{\sigma}\right)$ in the spin- $\frac{1}{2}$ representation, where $\theta_{e}, \theta_{j i} \in[0, \pi]$ are now the class angles of the group elements and $\hat{n}_{e}, \hat{n}_{j i} \in S^{2}$ unit vectors. Then, we may write

$$
Y_{e} \cdot \zeta_{\mathrm{S}}\left(H_{e^{*}}\left(h_{l}\right)\right)=\left|Y_{e}\right| \frac{\theta_{e}}{\kappa}\left(\frac{Y_{e}}{\left|Y_{e}\right|} \cdot \hat{n}_{e}\right), \quad X_{i j} \cdot \zeta\left(h_{j}^{-1} K_{j i}\left(h_{l}\right) h_{i}\right)=\left|X_{i j}\right| \frac{\theta_{j i}}{\kappa}\left(\frac{X_{i j}}{\left|X_{i j}\right|} \cdot \hat{n}_{j i}\right) .
$$

Considering then variations in the unit vectors $\hat{n}_{e}$ and $\hat{n}_{j i}$, it is immediate to find that the stationary phase of the amplitude is given by $\hat{n}_{e}= \pm \frac{Y_{e}}{\left|Y_{e}\right|} \cdot \hat{n}_{e}$ and $\hat{n}_{j i}= \pm \frac{X_{i j}}{\left|X_{i j}\right|}$, the signs corresponding to the two opposite orientations of the edge vectors or, equivalently, the dual faces. Now, if a configuration of edge vectors satisfies the constraints for a given discrete connection, it does so also for the oppositely oriented configuration obtained by reversing the orientations of all the dual faces. For the oppositely oriented configuration the holonomies around dual faces are also inverted, which gives opposite signs for $\hat{n}_{e}$ and $\hat{n}_{j i}$ with respect to the original configuration. Therefore, choosing $\hat{n}_{e}$ and $\hat{n}_{j i}$ to have positive signs for one of the orientations and thus negative signs for the other, we may further write for the Ponzano-Regge 
amplitude in the semi-classical limit

$$
\tilde{\mathcal{A}}_{\mathrm{PR}}^{\star \mathrm{lo}}\left(X_{i j}\right) \propto \int\left[\prod_{l} \mathrm{~d} h_{l}\right]\left[\prod_{e \notin \partial \Delta} \mathrm{d} Y_{e}\right] \cos \left(\frac{i}{\hbar \kappa}\left[\sum_{e \notin \partial \Delta}\left|Y_{e}\right| \theta_{e}+\sum_{\substack{(i, j) \in \mathcal{N} \\ i<j}}\left|X_{i j}\right| \theta_{j i}\right]\right),
$$

where the cosine arises from summing the contributions from both orientations of the triangulation. The argument of the cosine is exactly the first order Regge action, well-known from discrete gravity. Notice, however, that the deficit angles $\theta_{e}$ and the dihedral angles $\theta_{j i}$ still depend on the discrete bulk connection given by the group elements $h_{l}$, which are integrated over in the amplitude. Also, we have not yet imposed the closure constraints on the edge vectors, which arise from the variations of the bulk connection. These constraints impose the closure of edge vectors for each triangle, taking account orientations and parallel transports. At the same time they impose the metricity of the discrete connection and restrict the integrals over $Y_{e}$ to the geometric configurations, as in Regge gravity ${ }^{7}$. Solving for the discrete connection $h_{l}$ in terms of the edge vectors from the constraint equations (when possible, i.e., for non-degenerate configurations) leads to the second order Regge action and to the form

$$
\tilde{\mathcal{A}}_{\mathrm{PR}}^{\star l o}\left(X_{i j}\right) \propto\left[\prod_{e \notin \partial \Delta} \mathrm{d} Y_{e}\right] \cos \left(\frac{i}{\hbar \kappa}\left[\sum_{e \notin \partial \Delta}\left|Y_{e}\right| \theta_{e}+\sum_{\substack{(i, j) \in \mathcal{N} \\ i<j}}\left|X_{i j}\right| \theta_{j i}\right]\right)
$$

for the Ponzano-Regge amplitude, where now the deficit and dihedral angles are functions of the edge vectors, and only geometric configurations of the edge vectors are integrated over. Finally, we emphasize that for other choices of non-commutative structures, other than the one associated with the symmetric quantization map, we may obtain more complicated dependence on the dihedral and deficit angles. For example, the Freidel-Livine-Majid map leads to the compactified Regge action considered in [13,37]. However, all choices of non-commutative structures result in the same form as above in the regime of small deficit and dihedral angles. We thus see that the Regge action naturally arises in the semi-classical limit of the PonzanoRegge model in terms of the proper phase space variables.

Let us then move on to consider the 'on-shell' case, where we impose all the classical constraints on the path integral arising from the (non-commutative) stationary phase analysis. In this case the leading order semi-classical contribution to the Ponzano-Regge amplitude (5.6) reads in detail before integrating out the bulk variables

$$
\begin{aligned}
\tilde{\mathcal{A}}_{\mathrm{PR}}^{\star \mathrm{lo}}\left(X_{i j}\right) \propto & \int\left[\prod_{\substack{(i, j) \in \mathcal{N} \\
i<j}} \mathrm{~d} g_{i j} \mathrm{~d} g_{j i} c\left(g_{i j}^{-1} g_{j i}\right)\right]\left[\prod_{l} \mathrm{~d} h_{l}\right]\left[\prod_{e \notin \partial \Delta} \mathrm{d} X_{e}\right]\left[\prod_{e \notin \partial \Delta} \delta\left(H_{e^{*}}\left(h_{l}\right)\right)\right] \\
& \times\left[\prod_{\substack{i, j) \in \mathcal{N} \\
i<j}} \delta\left(g_{i j} h_{j}^{-1} K_{j i}\left(h_{l}\right) h_{i} g_{j i}^{-1}\right)\right]\left[\prod_{f \notin \partial \Delta} \delta_{\star}\left(\sum_{e \in f} \epsilon_{f e} \operatorname{Ad}_{h_{f e}} X_{e}\right)\right] \\
& \star\left[\prod_{i} \delta_{\star}\left(\sum_{\substack{(i, j) \in \mathcal{N} \\
j>i}} X_{i j}-\sum_{\substack{(i, j) \in \mathcal{N} \\
j<i}} \operatorname{Ad}_{g_{i j}^{-1} g_{j i}} X_{j i}\right)\right] \star \exp \left\{\frac{i}{\hbar} \sum_{\substack{(i, j) \in \mathcal{N} \\
i<j}} X_{i j} \cdot \zeta\left(g_{i j}^{-1} g_{j i}\right)\right\},
\end{aligned}
$$

where we have identified $Y_{i j}:=\operatorname{Ad}_{g_{i j}} X_{i j}=-\operatorname{Ad}_{g_{j i}} X_{j i}$ for all $(i, j) \in \mathcal{N}$ such that $i<j$. Here, the delta functions on the group impose triviality of the holonomies, which implies flatness of

\footnotetext{
${ }^{7}$ We note that for some choices of a quantization map, such as the Duflo map, the $\star$-delta function does not depend on a simple linear combination of its arguments, and thus the closure constraints must be non-linear outside the strict classical regime to match the exact result (4.7). However, for the symmetric quantization map we consider here the closure constraints remain undeformed in all orders.
} 
the discrete connection. The $\star$-delta functions impose closure of the edge vectors $e \in f$ belonging to the bulk and boundary triangles $f \in \Delta$, which in the discrete gravity context corresponds to the metricity of the discrete connection. The action is reduced due to the imposition of the flatness constraints to a simple boundary term.

Since the amplitude depends only on $G_{i j}$ and not the individual $g_{i j}$, we may further apply a change of variables by denoting $G_{i j}:=g_{j i}^{-1} g_{i j}$ and $G_{j i}=G_{i j}^{-1}$ for all $i<j$. These are the group elements that represent parallel transports between centers of boundary triangles, and are therefore naturally related to the dihedral angles of Regge calculus. By also integrating over the bulk variables, we obtain

$$
\begin{aligned}
\tilde{\mathcal{A}}_{\mathrm{PR}}^{\star \mathrm{lo}}\left(X_{i j}\right) \propto & {\left[\prod_{\substack{(i, j) \in \mathcal{N} \\
i<j}} \mathrm{~d} G_{i j} c\left(G_{i j}\right)\right]\left[\prod_{v \in \partial \Delta} \delta\left(H_{v}\left(G_{i j}\right)\right)\right] } \\
& \times\left[\prod_{i} \delta_{\star}\left(\sum_{\substack{(i, j) \in \mathcal{N} \\
j>i}} X_{i j}-\sum_{\substack{(i, j) \in \mathcal{N} \\
j<i}} \operatorname{Ad}_{G_{i j}}^{-1} X_{j i}\right)\right] \\
& \star \exp \left\{\frac{i}{\hbar} \sum_{\substack{i, j) \in \mathcal{N} \\
i<j}} X_{i j} \cdot \zeta\left(G_{i j}^{-1}\right)\right\} .
\end{aligned}
$$

The integrations over the bulk connection result in the delta functions imposing flatness of the boundary holonomies $H_{v}\left(G_{i j}\right)$ around all boundary vertices $v \in \partial \Delta$. In addition, the integrations over the bulk variables yield the volume of geometric bulk configurations, which contributes only to the normalization factor. The result of the calculation is exactly as we would expect from a first order $3 \mathrm{~d}$ discrete gravity action, namely, it is expressed as a function of edge vectors, where parallel transports are integrated over flat connections, which allow the edge vectors to satisfy the closure constraints.

One may further decompose the integrals in (5.9) over group elements $G_{i j}$ into integrals over dihedral class angles $\theta_{i j}:=\left|k\left(G_{i j}\right)\right|$, where $k(g):=-i \ln (g) \in \mathfrak{s u}(2) \cong \mathbb{R}^{3}$, and integrals over unit vectors $\hat{n}_{i j}:=k\left(G_{i j}\right) /\left|k\left(G_{i j}\right)\right| \in S^{2}$, such that $G_{i j} \equiv \exp \left(i \theta_{i j} \hat{n}_{i j} \cdot \vec{\sigma}\right)$ in the spin- $\frac{1}{2}$ representation. Adopting again the symmetric quantization map, we then have for the exponent

$$
\sum_{\substack{(i, j) \in \mathcal{N} \\ i<j}} X_{i j} \cdot \zeta_{\mathrm{S}}\left(G_{i j}^{-1}\right)=-\frac{1}{\kappa} \sum_{\substack{(i, j) \in \mathcal{N} \\ i<j}}\left|X_{i j}\right| \theta_{i j}\left(\frac{X_{i j}}{\left|X_{i j}\right|}\right) \cdot \hat{n}_{i j}
$$

The stationary phase with respect to the integrals over $\hat{n}_{i j} \in S^{2}$ is given by $\frac{X_{i j}}{\left|X_{i j}\right|} \cdot \hat{n}_{i j}= \pm 1$. Now the two solutions correspond to opposite orientations of the boundary, both contributing to the dominant phase with opposite signs, again turning the exponential into a cosine. Thus, we obtain

$$
\begin{aligned}
\tilde{\mathcal{A}}_{\mathrm{PR}}^{\mathrm{lo}}\left(X_{i j}\right) \propto & \int\left[\prod_{\substack{(i, j) \in \mathcal{N} \\
i<j}} \mathrm{~d} \theta_{i j}\left(\frac{\sin \theta_{i j}}{\theta_{i j}}\right)^{2}\right]\left[\prod_{v \in \partial \Delta} \delta\left(H_{v}\left(G_{i j}\right)\right)\right] \\
& \times\left[\prod_{i} \delta_{\star}\left(\sum_{\substack{(i, j) \in \mathcal{N} \\
j>i}} X_{i j}-\sum_{\substack{(i, j) \in \mathcal{N} \\
j<i}} \operatorname{Ad}_{G_{i j}}^{-1} X_{j i}\right)\right] \star \cos \left(\frac{i}{\hbar \kappa} \sum_{\substack{(i, j) \in \mathcal{N} \\
i<j}}\left|X_{i j}\right| \theta_{i j}\right),
\end{aligned}
$$

where $G_{i j} \equiv \exp \left(-i \theta_{i j}\left(X_{i j} /\left|X_{i j}\right|\right) \cdot \vec{\sigma}\right)$ and the $\left(\sin \theta_{i j} / \theta_{i j}\right)^{2}$ factors arise from the Haar measure. The second order expression may be obtained by solving the constraint equations for the 
boundary dihedral angles $\theta_{i j}$ in terms of the edge vectors $X_{i j}$, and substituting into the above formula. This leads to an expression of the form

$$
\tilde{\mathcal{A}}_{\mathrm{PR}}^{\mathrm{lo}}\left(X_{i j}\right) \propto \cos \left(\frac{i}{\hbar \kappa} \sum_{\substack{i, j \\ i<j}}\left|X_{i j}\right| \theta_{i j}\left(X_{k l}\right)\right) .
$$

This is the second order Regge action in terms of only the boundary metric data.

Thus, we have verified that the non-commutative variational method for the stationary phase approximation leads to the correct classical geometric constraints for the Ponzano-Regge model and agrees with the exact analysis of the amplitude. A similar result was obtained by us for the dual non-commutative representation of quantum mechanics on the group $\mathrm{SO}(3)$ previously in [45]. The use of such a non-commutative variational method may be motivated by noting that, in calculating transition amplitudes for boundary states, $\tilde{\mathcal{A}}_{\mathrm{PR}}\left(X_{i j}\right)$ acts as an integral kernel with respect to the $\star$-product, and not with respect to the commutative pointwise product. However, more work on this point is certainly needed in order to achieve a better understanding that would definitely settle the issue. Granting the use of the non-commutative stationary phase method, we have thus shown that the phase space path integral for the Ponzano-Regge model obtained through the non-commutative Fourier transform facilitates a straigthforward asymptotic analysis of the classical limit of the model. We also showed that the semi-classical approximation to the amplitude is given by the cosine of the Regge action, in agreement with previous studies [17].

Finally, we would like to comment on our saddle point analysis in comparison with the existing ones in the literature $[15,17]$. Besides not having a real-valued contribution to the exponent (that is, an imaginary contribution to the classical action), and thus having a simpler variational principle given by a standard (albeit non-commutative) saddle point approximation, we also do not have any logarithmic term. In other words, the flux representation of the spin foam model gives a discrete BF path integral in terms of the standard classical action. The choice of quantization map affects the exact form of such discrete classical action, but in a rather minor way. In fact, it leads to a discretization of the continuum curvature in terms of either the holonomy of the connection (a group element), for the FLM map, or its logarithm (a Lie algebra element), for the Duflo map. In general, the analysis appears to be more straightforward, than the one based on the spin (or coherent state) representation, as one would expect from a straightforward path integral representation. We will exemplify this comparison in the simple case of a triangulation formed by a single tetrahedron.

\section{Semi-classical limit for a tetrahedron}

To conclude our asymptotic analysis, we consider in this section the relation of the classical limit of Ponzano-Regge amplitudes in terms of non-commutative boundary metric variables to the usual formulation of spin foam asymptotics as the large spin limit in the spin representation. We will restrict our treatment to the case of a single tetrahedron, since in this case the asymptotics for the Ponzano-Regge amplitude is simple and well-known in the literature. In particular, it has been found that (for non-degenerate boundary data) the amplitude of a tetrahedron is approximated in the large spin limit by the cosine of the Regge action [17,36]. We derive this result from the asymptotic behavior obtained through the non-commutative phase space path integral, and thus establish a firm connection between the two asymptotic analyses.

For a single tetrahedron the Ponzano-Regge amplitude with boundary connection data reads

$$
\mathcal{A}_{\mathrm{PR}}\left(g_{i j}\right)=\int\left[\prod_{i} \mathrm{~d} h_{i}\right] \prod_{\substack{i, j \\ i<j}} \delta\left(g_{i j} h_{j}^{-1} h_{i} g_{j i}^{-1}\right)
$$


where $i, j=1, \ldots, 4$ label the boundary triangles, and the notation is chosen as in Fig. $2 .{ }^{8}$ The deltas impose flatness of the connection around all six wedges of the tetrahedra (see Fig. 1, where wedges are illustrated in grey). Now, this amplitude may be transformed into other representations described by other types of boundary data using different bases of functions on SU(2). In particular, as already explained above, one may transform the amplitude into the basis given by the non-commutative plane waves, in which case the amplitude is expressed as a function of non-commutative boundary edge vectors. Adopting for convenience the symmetric quantization map, this yields

$$
\begin{aligned}
\tilde{\mathcal{A}}_{\mathrm{PR}}\left(X_{i j}\right)= & \int\left[\prod_{i, j} \mathrm{~d} g_{i j} E\left(g_{i j}, X_{i j}\right)\right] \mathcal{A}_{\mathrm{PR}}\left(g_{i j}\right)=\int\left[\prod_{i, j} \mathrm{~d} g_{i j}\right]\left[\prod_{i} \mathrm{~d} h_{i}\right]\left[\prod_{\substack{i, j \\
i<j}} \mathrm{~d} Y_{j i}\right] \\
& \times \exp \left\{\frac{i}{\hbar}\left(\sum_{\substack{i, j \\
i<j}} Y_{i j} \cdot \zeta_{\mathrm{S}}\left(g_{i j} h_{j}^{-1} h_{i} g_{j i}^{-1}\right)-\sum_{i, j} X_{i j} \cdot \zeta_{\mathrm{S}}\left(g_{i j}\right)\right)\right\} .
\end{aligned}
$$

We emphasize that this is again nothing but the first order action for discrete $3 \mathrm{~d}$ gravity with boundary terms. Let us briefly sketch the asymptotic analysis for the Ponzano-Regge amplitude for a tetrahedron in non-commutative boundary metric variables. As before, by studying the non-commutative variations of the action

$$
\mathcal{S}_{\mathrm{PR}}=\sum_{\substack{i, j \\ i<j}} Y_{i j} \cdot \zeta_{\mathrm{S}}\left(g_{i j} h_{j}^{-1} h_{i} g_{j i}^{-1}\right)-\sum_{i, j} X_{i j} \cdot \zeta_{\mathrm{S}}\left(g_{i j}\right)
$$

we recover as stationary phase solutions in the classical limit the constraint equations

- Wedge flatness ${ }^{9}: g_{i j} h_{j}^{-1} h_{i} g_{j i}^{-1}=\mathbb{1}$ for all $i<j$,

- Identification of boundary edge vectors: $\operatorname{Ad}_{g_{i j}} X_{i j}=Y_{i j}=-\operatorname{Ad}_{g_{j i}} X_{j i}$ for all $i<j$, and

- Closure of the boundary edge vectors: $\sum_{j \neq i} \epsilon_{i j} X_{j i}=0$ for all $i$.

We then substitute these identities into the amplitude, whereby we find

$$
\begin{aligned}
\tilde{\mathcal{A}}_{\mathrm{PR}}^{\mathrm{lo}}\left(X_{i j}\right) \propto & \int\left[\prod_{i, j} \mathrm{~d} g_{i j}\right]\left[\prod_{v \in \partial \Delta} \delta\left(H_{v}\left(g_{i j}\right)\right)\right]\left[\prod_{i} \delta_{\star}\left(\sum_{j>i} X_{i j}-\sum_{j<i} \operatorname{Ad}_{g_{i j}^{-1} g_{j i}} X_{j i}\right)\right] \\
& \star \exp \left\{\frac{i}{\hbar} \sum_{\substack{i, j \\
i<j}} X_{i j} \cdot \zeta_{\mathrm{S}}\left(g_{i j}^{-1} g_{j i}\right)\right\}
\end{aligned}
$$

as the leading order contribution in $\hbar$ to the amplitude. Here, $\delta\left(H_{v}\left(g_{i j}\right)\right)$ impose flatness of holonomies around all boundary vertices $v \in \partial \Delta$ of the tetrahedron, which arise from the delta functions $\delta\left(g_{i j} h_{j}^{-1} h_{i} g_{j i}^{-1}\right)$ by integrating over all $h_{i}$. More precisely, we obtain the constraint only for three of the four vertices, which already imposes flatness for the fourth vertex as well. These conditions are nothing else than the Hamiltonian constraint of 3d gravity, generating the simplicial diffeomorphisms at each vertex of the triangulation (see, for example, [3, 5]). We may further apply a change of variables by denoting $G_{i j}:=g_{j i}^{-1} g_{i j}$ and $G_{j i}=G_{i j}^{-1}$ for all $i<j$. Again, these are the group elements that represent parallel transports between centers of boundary triangles, and are therefore naturally related to the dihedral angles of Regge calculus.

\footnotetext{
${ }^{8}$ Of course, in the case of a single tetrahedron $K_{i j}=\mathbb{1}$ for all $i, j$, since the paths along which $K_{i j}$ parallel transport (as in Fig. 2) are of length 0.

${ }^{9}$ This is basically the condition enforcing the piece-wise flat situation, i.e., the flatness of the tetrahedron.
} 
As before in the more general case, one may further decompose the integrals over group elements $G_{i j}$ into integrals over dihedral class angles $\theta_{i j}:=\kappa\left|\zeta_{\mathrm{S}}\left(G_{i j}\right)\right|$ and integrals over unit vectors $\hat{n}_{i j}:=\zeta_{\mathrm{S}}\left(G_{i j}\right) /\left|\zeta_{\mathrm{S}}\left(G_{i j}\right)\right| \in S^{2}$. The stationary phase equations for the unit vectors $\hat{n}_{i j}$ lead to the expression

$$
\begin{aligned}
& \tilde{\mathcal{A}}_{\mathrm{PR}}^{\mathrm{lo}}\left(X_{i j}\right) \propto \int\left[\prod_{\substack{i, j \\
i<j}} \mathrm{~d} \theta_{i j}\left(\frac{\sin \theta_{i j}}{\theta_{i j}}\right)^{2}\right]\left[\prod_{v \in \partial \Delta} \delta\left(H_{v}\left(G_{i j}\right)\right)\right]\left[\prod_{i} \delta_{\star}\left(\sum_{j>i} X_{i j}-\sum_{j<i} \mathrm{Ad}_{G_{i j}}^{-1} X_{j i}\right)\right] \\
& \star \cos \left(\frac{i}{\hbar \kappa} \sum_{\substack{i, j \\
i<j}}\left|X_{i j}\right| \theta_{i j}\right)
\end{aligned}
$$

which is just the formula (5.10) for a single tetrahedron. The argument of the cosine in (6.3) is the first order Regge action for a tetrahedron.

As the Regge action has previously been recovered in the semi-classical limit of the PonzanoRegge model in the spin representation by using coherent states, we wish to link our calculation to the spin basis, which is given by the $\mathrm{SU}(2)$ Wigner $D$-matrices $D_{k l}^{j}(g)$. We find for the Ponzano-Regge amplitude in the spin basis

$$
\begin{aligned}
\tilde{\mathcal{A}}_{\mathrm{PR}}\left(j_{i j} ; k_{i j}, l_{i j}\right)= & \int\left[\prod_{i, j} \mathrm{~d} g_{i j} D_{k_{i j} l_{i j}}^{j_{i j}}\left(g_{i j}\right)\right] \mathcal{A}_{\mathrm{PR}}\left(g_{i j}\right) \\
= & \left\{\begin{array}{lll}
j_{12} & j_{13} & j_{14} \\
j_{23} & j_{24} & j_{34}
\end{array}\right\}\left[\prod_{i<j} \delta^{j_{i j} j_{j i}} \delta_{k_{i j} k_{j i}}\right]\left(\begin{array}{lll}
j_{21} & j_{31} & j_{41} \\
l_{21} & l_{31} & l_{41}
\end{array}\right)\left(\begin{array}{ccc}
j_{21} & j_{32} & j_{42} \\
-l_{12} & l_{32} & l_{42}
\end{array}\right) \\
& \times\left(\begin{array}{ccc}
j_{31} & j_{32} & j_{43} \\
-l_{13} & -l_{23} & l_{43}
\end{array}\right)\left(\begin{array}{ccc}
j_{41} & j_{42} & j_{43} \\
-l_{14} & -l_{24} & -l_{34}
\end{array}\right),
\end{aligned}
$$

where we introduced the $\mathrm{SU}(2) 6 j$-symbol and the $3 j m$-symbol, familiarly denoted as

$$
\left\{\begin{array}{lll}
j_{1} & j_{2} & j_{3} \\
j_{4} & j_{5} & j_{6}
\end{array}\right\} \quad \text { and } \quad\left(\begin{array}{ccc}
j_{1} & j_{2} & j_{3} \\
m_{1} & m_{2} & m_{3}
\end{array}\right)
$$

respectively, which are the basic building blocks of Ponzano-Regge model in the spin representation. Typically, to study the asymptotics of spin foams one considers the formula for the square of the $6 j$-symbol expressed in terms of integrals over $\mathrm{SU}(2)$ characters $\chi^{j}: \mathrm{SU}(2) \rightarrow \mathbb{C}$ as

$$
\left\{\begin{array}{lll}
j_{12} & j_{13} & j_{14} \\
j_{23} & j_{24} & j_{34}
\end{array}\right\}^{2}=\int\left[\prod_{i, j} \mathrm{~d} g_{i j}\right]\left[\prod_{i} \mathrm{~d} h_{i}\right]\left[\prod_{i<j} \chi^{j_{i j}}\left(g_{i j} h_{j}^{-1} h_{i} g_{j i}^{-1}\right)\right],
$$

where $g_{i j}$ correspond to boundary connection variables, and $h_{i}$ correspond to parallel transports from the boundary triangles to the center of the tetrahedron, as before. This corresponds to the Ponzano-Regge amplitude for a tetrahedron with fixed quantized edge lengths, since by fixing the boundary connection $g_{i j}$ and summing over all $j_{i j}$ (with weights $\left(2 j_{i j}+1\right)$ ) we arrive again at (6.1). More accurately, from (6.4) we find

$$
\left\{\begin{array}{lll}
j_{12} & j_{13} & j_{14} \\
j_{23} & j_{24} & j_{34}
\end{array}\right\}^{2}=\sum_{\substack{j_{j i} \\
i<j}} \sum_{k_{i j}, l_{i j}}\left[\prod_{i<j} \delta_{l_{i j} l_{j i}}\right] \tilde{\mathcal{A}}_{\mathrm{PR}}\left(j_{i j} ; k_{i j}, l_{i j}\right) .
$$

Thus, denoting still by $\tilde{\mathcal{A}}_{\mathrm{PR}}\left(j_{i j} ; k_{i j}, l_{i j}\right)$ the amplitude, where we have set $j_{j i}=j_{i j}, k_{i j}=k_{j i}$ and $l_{i j}=l_{j i}$, we may write

$$
\left\{\begin{array}{lll}
j_{12} & j_{13} & j_{14} \\
j_{23} & j_{24} & j_{34}
\end{array}\right\}^{2}=\sum_{k_{i j}, l_{i j}} \tilde{\mathcal{A}}_{\mathrm{PR}}\left(j_{i j} ; k_{i j}, l_{i j}\right)
$$


Let us define functions

$$
D_{\hat{m} \hat{n}}^{j}(g):=\left\langle j, \hat{m}\left|D^{j}(g)\right| j, \hat{n}\right\rangle \equiv\left\langle\frac{1}{2}, \hat{m}\left|D^{\frac{1}{2}}(g)\right| \frac{1}{2}, \hat{n}\right\rangle^{2 j}
$$

where $D^{j}(g)$ are the $\mathrm{SU}(2)$ Wigner matrices of spin- $j$ representation, and $|j, \hat{m}\rangle$ are Perelomov coherent states [46] on $\mathrm{SU}(2)$ labelled by a representation $j \in\left\{\frac{n}{2}: n \in \mathbb{N}\right\}$ and a unit vector $\hat{m} \in S^{2}$. By applying the decomposition of unity in terms of the Perelomov coherent states

$$
(2 j+1) \int_{S^{2}} \frac{\mathrm{d} \hat{m}}{4 \pi}|j, \hat{m}\rangle\langle j, \hat{m}|=\mathbb{1}_{j},
$$

it is easy to show that $D_{\hat{m} \hat{n}}^{j}(g)$ constitute an over-complete basis of functions on $\mathrm{SU}(2)$, as we have

$$
\delta\left(g^{-1} g^{\prime}\right)=\sum_{j}(2 j+1)^{3} \int_{S^{2}} \frac{\mathrm{d} \hat{m}}{4 \pi} \int_{S^{2}} \frac{\mathrm{d} \hat{n}}{4 \pi} \overline{D_{\hat{m} \hat{n}}^{j}(g)} D_{\hat{m} \hat{n}}^{j}\left(g^{\prime}\right) .
$$

Similarly, for the $\mathrm{SU}(2)$ character function we may write

$$
\chi^{j}(g)=\operatorname{tr}\left(D^{j}(g)\right)=(2 j+1) \int_{S^{2}} \frac{\mathrm{d} \hat{m}}{4 \pi} D_{\hat{m} \hat{m}}^{j}(g) .
$$

In the following, without a serious danger of confusion, we will denote the spin- $\frac{1}{2}$ representation Wigner matrices and coherent states simply by $D^{\frac{1}{2}}(g)=: g$ and $\left|\frac{1}{2}, \hat{m}\right\rangle=:|\hat{m}\rangle$, respectively, so in our notation $D_{\hat{m} \hat{n}}^{j}(g) \equiv\langle\hat{m}|g| \hat{n}\rangle^{2 j}$. Using (6.7), the $6 j$-symbol (6.5) may be re-expressed as

$$
\left\{\begin{array}{lll}
j_{12} & j_{13} & j_{14} \\
j_{23} & j_{24} & j_{34}
\end{array}\right\}^{2}=\left[\prod_{i, j}\left(2 j_{i j}+1\right)^{2} \int \frac{\mathrm{d} \hat{m}_{i j}}{4 \pi} \frac{\mathrm{d} \hat{n}_{i j}}{4 \pi}\right] \hat{\mathcal{A}}_{\mathrm{PR}}\left(j_{i j} ; \hat{m}_{i j}, \hat{n}_{i j}\right) .
$$

This is simply the equation (6.6) transformed into the coherent state basis. Here, $\hat{\mathcal{A}}_{\mathrm{PR}}\left(j_{i j} ; \hat{m}_{i j}\right.$, $\left.\hat{n}_{i j}\right)$ is indeed the Ponzano-Regge amplitude for a single tetrahedron with the coherent state labels as boundary metric data, which may be written as

$$
\begin{aligned}
& \hat{\mathcal{A}}_{\mathrm{PR}}\left(j_{i j} ; \hat{m}_{i j}, \hat{n}_{i j}\right)=\int\left[\prod_{i, j} \mathrm{~d} g_{i j} D_{\hat{m}_{i j} \hat{n}_{i j}}^{j_{i j}}\left(g_{i j}\right)\right] \mathcal{A}_{\mathrm{PR}}\left(g_{i j}\right) \\
& =\left\{\begin{array}{lll}
j_{12} & j_{13} & j_{14} \\
j_{23} & j_{24} & j_{34}
\end{array}\right\}\left[\prod_{\substack{i, j \\
i<j}} \delta_{j_{i j} j_{j i}} \delta_{\hat{m}_{i j},-\hat{m}_{j i}}^{j_{i j}}\right] \prod_{i}\left(\begin{array}{lll}
j_{i j_{1}} & j_{i j_{2}} & j_{i j_{3}} \\
\hat{n}_{i j_{1}} & \hat{n}_{i j_{2}} & \hat{n}_{i j_{3}}
\end{array}\right),
\end{aligned}
$$

where we denote $\delta_{\hat{m}_{i j},-\hat{m}_{j i}}^{j_{i j}}:=\left\langle\hat{m}_{i j} \mid-\hat{m}_{j i}\right\rangle^{2 j_{i j}}$, and

$$
\left(\begin{array}{lll}
j_{1} & j_{2} & j_{3} \\
\hat{n}_{1} & \hat{n}_{2} & \hat{n}_{3}
\end{array}\right):=\sum_{k_{i}}\left(\begin{array}{lll}
j_{1} & j_{2} & j_{3} \\
k_{1} & k_{2} & k_{3}
\end{array}\right)\left\langle j_{1}, k_{1} \mid j_{1}, \hat{n}_{1}\right\rangle\left\langle j_{2}, k_{2} \mid j_{2}, \hat{n}_{2}\right\rangle\left\langle j_{3}, k_{3} \mid j_{3}, \hat{n}_{3}\right\rangle
$$

is the Wigner $3 j m$-symbol in the coherent state basis.

On the other hand, we may write the same amplitude (6.9), transformed from the noncommutative variables, as

$$
\hat{\mathcal{A}}_{\mathrm{PR}}\left(j_{i j} ; \hat{m}_{i j}, \hat{n}_{i j}\right)=\int\left[\prod_{\substack{i, j \\ i<j}} \frac{\mathrm{d} X_{i j}}{(2 \pi \hbar)^{3}} \widetilde{D_{\hat{m}_{i j} \hat{n}_{i j}}^{j_{i j}}}\left(X_{i j}\right)\right] \star \tilde{\mathcal{A}}_{\mathrm{PR}}\left(X_{i j}\right),
$$


where, adopting for convenience the non-commutative structure associated to the symmetric quantization map, we denote by

$$
\widetilde{D_{\hat{m} \hat{n}}^{j}}(X):=\int \frac{\mathrm{d} g}{\kappa^{3}} \overline{E(g, X)} D_{\hat{m} \hat{n}}^{j}(g)=\int \frac{\mathrm{d} g}{\kappa^{3}} \exp \left\{\frac{i}{\hbar}\left[-2 i \hbar j \ln \langle\hat{m}|g| \hat{n}\rangle-\zeta_{\mathrm{S}}(g) \cdot X\right]\right\}
$$

the non-commutative Fourier transform of $D_{\hat{m} \hat{n}}^{j}(g)$. Now, let us consider the stationary phase approximation of the expression (6.10). The exponential in (6.11) has real and imaginary parts, both of which must be taken into account in the stationary phase approximation. For the noncommutative stationary phase equations of this expression in the classical limit $\hbar \rightarrow 0, j \rightarrow \infty$, $\hbar j=$ const, we obtain by a straightforward calculation $\hat{n}=\operatorname{Ad}_{g} \hat{m}$ and $2 \hbar j \hat{m}=\frac{1}{\kappa} X$. Note that, since we had already understood the $X$ variables as the classical discrete BF variables, this result confirms that the coherent state variables acquire the correct geometric interpretation in the classical limit.

Then, from (6.2) and $2 \hbar \kappa j_{i j} \hat{m}_{i j}=X_{i j}, \hat{n}_{i j}=\operatorname{Ad}_{g_{i j}} \hat{m}_{i j}$, we have for the classical limit of (6.10) the expression

$$
\begin{aligned}
\hat{\mathcal{A}}_{\mathrm{PR}}\left(j_{i j} ; \hat{m}_{i j}, \hat{n}_{i j}\right) \propto & \int\left[\prod_{i, j} \mathrm{~d} g_{i j}\right]\left[\prod_{v \in \partial \Delta} \delta\left(H_{v}\left(g_{i j}\right)\right)\right] \\
& \times\left[\prod_{i} \delta\left(\sum_{j>i} j_{i j} \hat{m}_{i j}-\sum_{j<i} \operatorname{Ad}_{g_{i j}^{-1} g_{j i}} j_{j i} \hat{m}_{j i}\right)\right] \\
& \times\left[\prod_{\substack{i, j) \in \mathcal{N} \\
i<j}} \delta\left(\hat{n}_{i j}-\operatorname{Ad}_{g_{i j}} \hat{m}_{i j}\right)\right] \exp \left\{\frac{i}{\hbar} \sum_{\substack{i, j \\
i<j}} 2 \hbar j_{i j} \hat{m}_{i j} \cdot \zeta_{\mathrm{s}}\left(g_{i j}^{-1} g_{j i}\right)\right\} \\
& \times(1+\mathcal{O}(\hbar)),
\end{aligned}
$$

where the variation with respect to $X_{i j}$ gives the identification of the group elements of the two asymptotic expressions. Equating the above with the classical limit of the expression (6.9) and integrating on both sides over all $\hat{m}_{i j}, \hat{n}_{i j}$ as in (6.8) yields

$$
\left\{\begin{array}{lll}
j_{12} & j_{13} & j_{14} \\
j_{23} & j_{24} & j_{34}
\end{array}\right\}^{2} \propto \int\left[\prod_{\substack{(i, j) \in \mathcal{N} \\
i<j}} \mathrm{~d} \hat{m}_{i j} \mathrm{~d} G_{i j}\right] \exp \left\{\frac{i}{\hbar} \sum_{\substack{(i, j) \in \mathcal{N} \\
i<j}} 2 \hbar j_{i j} \hat{m}_{i j} \cdot \zeta_{\mathrm{S}}\left(G_{i j}\right)\right\}(1+\mathcal{O}(\hbar)),
$$

where the integral is over sets of unit vectors $\left\{\hat{m}_{i j}\right\}$ such that the edge vectors $2 \hbar \kappa j_{i j} \hat{m}_{i j}$ satisfy the closure constraints for each triangle $f_{i} \in \partial \Delta$ up to parallel transports given by $G_{i j}$. As above leading to (6.3) and the first order Regge action, we may further decompose the integrals over $G_{i j}$ into integrals over dihedral class angles and unit vectors. The stationary phase of the exponential of the above amplitude in this limit is again given by the first order Regge action

$$
S_{\text {Regge }}=\sum_{\substack{(i, j) \in \mathcal{N} \\ i<j}} 2 \hbar j_{i j} \theta_{i j}, \quad \theta_{i j} \in(-\pi, \pi)
$$

but now in terms of the coherent state variables. In essence, the result follows simply because in the classical limit the combination $2 \hbar \kappa j_{i j} \hat{m}_{i j}$ of coherent state variables gets identified with the true phase space variables, the edge vectors $X_{i j}$. This identification is due to the asymptotic behavior of the function $\widetilde{D_{\hat{m} \hat{n}}^{j}}(X)$ defined in (6.11), which mediates the transformation between the coherent state basis and the non-commutative basis. 


\section{Conclusions and comments}

Let us then summarize our results. We applied the non-commutative Fourier transform to express the Ponzano-Regge spin foam amplitude as a first order phase space path integral, which took the form of a discrete BF theory with standard classical action in terms of noncommutative metric boundary data. The choice of the quantization map for the geometric observables was seen to be intimately connected to the choice of a discretization for the $3 \mathrm{~d}$ BF theory. The path integral reformulation then allowed us to study conveniently the classical approximation to the full amplitude.

We discovered that depending on the choice of a non-commutative structure arising from the deformation quantization applied to the geometric observables, different limiting behaviors appear for the boundary data in the classical limit, when we apply the ordinary 'commutative' variational calculus to find the stationary phase solutions. Furthermore, in this case, the constraints that arise as the classical equations of motion generically do not correspond to discrete geometries, since the edge vectors in the constraint equations are deformed due to the nonlinearity of the group manifold. We verified our observation by considering as explicit examples the non-commutative structures that arise from symmetric, Duflo and Freidel-Livine-Majid quantization maps.

Accordingly, we were led to consider a non-commutative variational method to extract the stationary phase behavior, which was motivated by the fact that the amplitude for non-commutative metric boundary data acts as the integral kernel in the propagator with respect to the corresponding $\star$-product, and not the commutative product. We showed that the non-commutative variations produce the correct geometric constraints for the discrete metric boundary data in the classical limit. Thus, we concluded that only by taking into account the deformation of phase space structure in studying the variations, we find the undeformed and unambiguous geometric constraints, independent on the choice of the quantization map.

Finally, we considered the asymptotics of the $\mathrm{SU}(2) 6 j$-symbol, which is related to the Ponzano-Regge amplitude for a tetrahedra with fixed quantized edge lengths. We found the Regge action, previously recovered in the large spin limit of the $6 j$-symbol, in the classical limit. Our calculations thus not only verify the previous results, but also allows for a better understanding of them due to the clear-cut connection to the phase space of classical discretized $3 \mathrm{~d}$ gravity. This concrete example also illustrates the use of the non-commutative path integral as a computational tool. On the other hand, the full agreement of the results obtained via the non-commutative method with those obtained via ordinary commutative stationary phase method in the coherent state representation further validates the use of the non-commutative variations in extracting the asymptotic behavior of the amplitude in the classical limit.

There are several conclusions and further directions of research pointed to by our results. First and foremost, we have seen that the non-commutative metric representation obtained through the non-commutative Fourier transform facilitates a full asymptotic analysis of spin foam models, when proper care is taken in applying variational methods to the first order path integral. In particular, by studying the non-commutative variations one may recover the classical geometric constraints for all cases of non-commutative structures. The need for a noncommutative variational method requires further analysis, and must be taken into account in any future application of the non-commutative methods to spin foam models. Our consideration of the $6 j$-symbol asymptotics further illustrates the usefulness of the non-commutative methods.

As the non-commutative Fourier transform formalism has recently been extended to all exponential Lie groups [27], in particular the double-cover $\mathrm{SL}(2, \mathbb{C})$ of the Lorentz group, we look forward to extending the asymptotic analysis to the $4 \mathrm{~d}$ spin foam models in future work, now equipped with the improved understanding of the methods involved. In particular, it will be interesting to see how the simplicity constraints turn out to be imposed on the non-commutative 
metric variables in the phase space path integral measure for the different spin foam models proposed in the 4-dimensional case. The current formulation of $4 \mathrm{~d}$ models uses the non-commutative Fourier transform for the $\mathrm{BF}$ variables (based on $\mathrm{SO}(4)$, but extendable to Lorentzian models with $\mathrm{SL}(2, \mathbb{C}))$, on which the simplicity constraints are imposed. The advantages of the noncommutative formulation are twofold: First, the simplicity constraints may be imposed in a very geometrically transparent and natural way via insertions of non-commutative delta functions in the amplitudes, as one would expect. Secondly, in such variables, the spin foam amplitudes take again, as in the $3 \mathrm{~d}$ case, the explicit form of simplicial gravity path integrals. As we have shown in this paper, this will greatly facilitate the semi-classical analysis, which would proceed in entirely the same fashion as the one performed here. Such an analysis may help to elucidate the differences between the geometric properties of the current $4 \mathrm{~d}$ models, and even to propose new models with improved geometric behavior in the semi-classical limit.

\section{A Infinitesimal Baker-Campbell-Hausdorff formula}

In order to calculate the deformation matrix (5.7) for the symmetric quantization map, we need to compute Lie derivatives $\tilde{\mathcal{L}}_{k} \zeta_{\mathrm{s}, l}$ of the coordinates $\zeta_{\mathrm{S}}=-\frac{i}{\kappa} \ln (g)$ on $\mathrm{SU}(2)$. To do this, we derive the explicit form of the Baker-Campbell-Hausdorff formula $B\left(k, k^{\prime}\right)$ in $e^{i k \cdot \vec{\sigma}} e^{i k^{\prime} \cdot \vec{\sigma}} \equiv e^{i B\left(k, k^{\prime}\right) \cdot \vec{\sigma}}$ for the case, when one of the arguments is infinitesimal. We may write

$$
\begin{aligned}
\cos \mid B( & \left.k, k^{\prime}\right) \mid \mathbb{1}+i \frac{\sin \left|B\left(k, k^{\prime}\right)\right|}{\left|B\left(k, k^{\prime}\right)\right|} B\left(k, k^{\prime}\right) \cdot \vec{\sigma} \\
= & \left(\cos |k| \mathbb{1}+i \frac{\sin |k|}{|k|} k \cdot \vec{\sigma}\right)\left(\cos \left|k^{\prime}\right| \mathbb{1}+i \frac{\sin \left|k^{\prime}\right|}{\left|k^{\prime}\right|} k^{\prime} \cdot \vec{\sigma}\right) \\
= & \left(\cos |k| \cos \left|k^{\prime}\right|-\frac{\sin |k|}{|k|} \frac{\sin \left|k^{\prime}\right|}{\left|k^{\prime}\right|}\left(k \cdot k^{\prime}\right)\right) \mathbb{1} \\
& +i\left(\cos \left|k^{\prime}\right| \frac{\sin |k|}{|k|} k+\cos |k| \frac{\sin \left|k^{\prime}\right|}{\left|k^{\prime}\right|} k^{\prime}-\frac{\sin |k|}{|k|} \frac{\sin \left|k^{\prime}\right|}{\left|k^{\prime}\right|}\left(k \wedge k^{\prime}\right)\right) \cdot \vec{\sigma},
\end{aligned}
$$

where by $\wedge$ we denote the cross-product in $\mathbb{R}^{3}$, from which one can extract

$$
\begin{aligned}
& \cos \left|B\left(k, k^{\prime}\right)\right|=\cos |k| \cos \left|k^{\prime}\right|-\frac{\sin |k|}{|k|} \frac{\sin \left|k^{\prime}\right|}{\left|k^{\prime}\right|}\left(k \cdot k^{\prime}\right), \\
& \frac{\sin \left|B\left(k, k^{\prime}\right)\right|}{\left|B\left(k, k^{\prime}\right)\right|} B\left(k, k^{\prime}\right)=\cos \left|k^{\prime}\right| \frac{\sin |k|}{|k|} k+\cos |k| \frac{\sin \left|k^{\prime}\right|}{\left|k^{\prime}\right|} k^{\prime}-\frac{\sin |k|}{|k|} \frac{\sin \left|k^{\prime}\right|}{\left|k^{\prime}\right|}\left(k \wedge k^{\prime}\right) .
\end{aligned}
$$

From these identities it is not too difficult to find a closed form for the Baker-CampbellHausdorff formula for $\mathrm{SU}(2)$ in terms of elementary functions [27]. However, we will only need to consider the special case $k^{\prime}=t e_{k}$, where $t>0$ is an expansion parameter, and $e_{k}$, $k=1,2,3$, are orthonormal basis vectors in $\mathbb{R}^{3}$. Then we obtain the deformation matrix as $D_{k l}^{\mathrm{S}}(g)=\tilde{\mathcal{L}}_{k} \zeta_{\mathrm{s}, l}(g)=\left.\frac{\mathrm{d}}{\mathrm{d} t} B\left(k(g), t e_{k}\right)_{l}\right|_{t=0}$, i.e., it is the $t$-linear term in $B\left(k(g), t e_{k}\right)_{l}$. From above we have

$$
\frac{\sin \left|B\left(k, t e_{k}\right)\right|}{\left|B\left(k, t e_{k}\right)\right|} B\left(k, t e_{k}\right)_{l}=\frac{\sin |k|}{|k|} k_{l}+t\left(\cos |k| \delta_{k l}-\left(k \wedge e_{k}\right)_{l}\right)+\mathcal{O}\left(t^{2}\right),
$$

from which we may deduce

$$
\begin{aligned}
& \left|B\left(k, t e_{k}\right)\right|=|k|+t \frac{k_{k}}{|k|}+\mathcal{O}\left(t^{2}\right), \\
& \frac{1}{\sin \left|B\left(k, t e_{k}\right)\right|}=\frac{1}{\sin |k|}\left(1-t \frac{\cos |k|}{\sin |k|} \frac{k_{k}}{|k|}\right)+\mathcal{O}\left(t^{2}\right) .
\end{aligned}
$$


Using these formulae, we get

$$
B\left(k, t e_{k}\right)_{l}=k_{l}+t \frac{|k|}{\sin |k|}\left[\cos |k| \delta_{k l}+\left(\frac{\sin |k|}{|k|}-\cos |k|\right) \frac{k_{k} k_{l}}{|k|^{2}}-\epsilon_{k l}{ }^{m} k_{m}\right]+\mathcal{O}\left(t^{2}\right),
$$

and so

$$
D_{k l}^{\mathrm{S}}(g)=\frac{|k(g)|}{\sin |k(g)|}\left[\cos |k(g)| \delta_{k l}+\left(\frac{\sin |k(g)|}{|k(g)|}-\cos |k(g)|\right) \frac{k_{k}(g) k_{l}(g)}{|k(g)|^{2}}-\epsilon_{k l}{ }^{m} k_{m}(g)\right] .
$$

\section{Acknowledgments}

We are grateful for the anonymous referees for their constructive questions and comments, which led to several improvements to the original manuscript. We would like to thank A. Baratin for several useful discussions on the non-commutative Fourier transform and spin foam models. We also thank C. Guedes, F. Hellmann and W. Kaminski for several discussions. This work was supported by the A. von Humboldt Stiftung, through a Sofja Kovalevskaja Prize, which is gratefully acknowledged. The work of M. Raasakka was partially supported by Emil Aaltonen Foundation.

\section{References}

[1] Alexandrov S., Geiller M., Noui K., Spin foams and canonical quantization, SIGMA 8 (2012), 055, 79 pages, arXiv:1112.1961.

[2] Baez J.C., An introduction to spin foam models of $B F$ theory and quantum gravity, in Geometry and Quantum Physics (Schladming, 1999), Lecture Notes in Phys., Vol. 543, Springer, Berlin, 2000, 25-93, gr-qc/9905087.

[3] Bahr B., Dittrich B., (Broken) gauge symmetries and constraints in Regge calculus, Classical Quantum Gravity 26 (2009), 225011, 34 pages, arXiv:0905.1670.

[4] Baratin A., Dittrich B., Oriti D., Tambornino J., Non-commutative flux representation for loop quantum gravity, Classical Quantum Gravity 28 (2011), 175011, 19 pages, arXiv:1004.3450.

[5] Baratin A., Girelli F., Oriti D., Diffeomorphisms in group field theories, Phys. Rev. D 83 (2011), 104051, 22 pages, arXiv:1101.0590.

[6] Baratin A., Oriti D., Group field theory with noncommutative metric variables, Phys. Rev. Lett. 105 (2010), 221302, 4 pages, arXiv:1002.4723.

[7] Baratin A., Oriti D., Quantum simplicial geometry in the group field theory formalism: reconsidering the Barrett-Crane model, New J. Phys. 13 (2011), 125011, 28 pages, arXiv:1108.1178.

[8] Baratin A., Oriti D., Group field theory and simplicial gravity path integrals: a model for Holst-Plebański gravity, Phys. Rev. D 85 (2012), 044003, 15 pages, arXiv:1111.5842.

[9] Barrett J.W., Crane L., Relativistic spin networks and quantum gravity, J. Math. Phys. 39 (1998), 32963302, gr-qc/9709028,.

[10] Barrett J.W., Dowdall R.J., Fairbairn W.J., Hellmann F., Pereira R., Lorentzian spin foam amplitudes: graphical calculus and asymptotics, Classical Quantum Gravity 27 (2010), 165009, 34 pages, arXiv:0907.2440.

[11] Barrett J.W., Naish-Guzman I., The Ponzano-Regge model, Classical Quantum Gravity 26 (2011), 155014, 48 pages, arXiv:0803.3319.

[12] Boulatov D.V., A model of three-dimensional lattice gravity, Modern Phys. Lett. A 7 (1992), 1629-1646, hep-th/9202074.

[13] Caselle M., D'Adda A., Magnea L., Regge calculus as a local theory of the Poincaré group, Phys. Lett. B 232 (1989), 457-461.

[14] Chaichian M., Demichev A., Path integrals in physics. Vol. I. Stochastic processes and quantum mechanics, Series in Mathematical and Computational Physics, Institute of Physics Publishing, Bristol, 2001. 
[15] Conrady F., Freidel L., Semiclassical limit of 4-dimensional spin foam models, Phys. Rev. D 78 (2008), 104023, 18 pages, arXiv:0809.2280.

[16] Dittrich B., Guedes C., Oriti D., On the space of generalized fluxes for loop quantum gravity, Classical Quantum Gravity 30 (2013), 055008, 24 pages, arXiv:1205.6166.

[17] Dowdall R.J., Gomes H., Hellmann F., Asymptotic analysis of the Ponzano-Regge model for handlebodies, J. Phys. A: Math. Theor. 43 (2010), 115203, 27 pages, arXiv:0909.2027.

[18] Dupuis M., Girelli F., Livine E., Spinors and Voros star-product for group field theory: first contact, Phys. Rev. D 86 (2012), 105034, 18 pages, arXiv:1107.5693.

[19] Dupuis M., Livine E.R., Holomorphic simplicity constraints for 4D spinfoam models, Classical Quantum Gravity 28 (2011), 215022, 32 pages, arXiv:1104.3683.

[20] Engle J., Livine E., Pereira R., Rovelli C., LQG vertex with finite Immirzi parameter, Nuclear Phys. B 799 (2008), 136-149, arXiv:0711.0146.

[21] Engle J., Pereira R., Rovelli C., Loop-quantum-gravity vertex amplitude, Phys. Rev. Lett. 99 (2007), 161301, 4 pages, arXiv:0705.2388.

[22] Freidel L., Group field theory: an overview, Internat. J. Theoret. Phys. 44 (2005), 1769-1783, hep-th/0505016.

[23] Freidel L., Krasnov K., A new spin foam model for 4D gravity, Classical Quantum Gravity 25 (2008), 125018, 36 pages, arXiv:0708.1595.

[24] Freidel L., Livine E.R., 3D quantum gravity and effective noncommutative quantum field theory, Phys. Rev. Lett. 96 (2006), 221301, 4 pages, hep-th/0512113.

[25] Freidel L., Majid S., Noncommutative harmonic analysis, sampling theory and the Duflo map in $2+1$ quantum gravity, Classical Quantum Gravity 25 (2008), 045006, 37 pages, hep-th/0601004.

[26] Goldman W.M., The symplectic nature of fundamental groups of surfaces, Adv. Math. 54 (1984), 200-225.

[27] Guedes C., Oriti D., Raasakka M., Quantization maps, algebra representation, and non-commutative Fourier transform for Lie groups, J. Math. Phys. 54 (2013), 083508, 31 pages, arXiv:1301.7750.

[28] Han M., On spinfoam models in large spin regime, Classical Quantum Gravity 31 (2013), 015004, 21 pages, arXiv:1304.5627.

[29] Han M., Semiclassical analysis of spinfoam model with a small Barbero-Immirzi parameter, Phys. Rev. D 88 (2013), 044051, 13 pages, arXiv:1304.5628.

[30] Han M., Krajewski T., Path integral representation of Lorentzian spinfoam model, asymptotics and simplicial geometries, Classical Quantum Gravity 31 (2014), 015009, 34 pages, arXiv:1304.5626.

[31] Han M., Zhang M., Asymptotics of the spin foam amplitude on simplicial manifold: Euclidean theory, Classical Quantum Gravity 29 (2012), 165004, 40 pages, arXiv:1109.0500.

[32] Han M., Zhang M., Asymptotics of spinfoam amplitude on simplicial manifold: Lorentzian theory, Classical Quantum Gravity 30 (2013), 165012, 57 pages, arXiv:1109.0499.

[33] Hellmann F., Kamiński W., Geometric asymptotics for spin foam lattice gauge gravity on arbitrary triangulations, arXiv:1210.5276.

[34] Hellmann F., Kamiński W., Holonomy spin foam models: asymptotic geometry of the partition function, J. High Energy Phys. 2013 (2013), no. 10, 165, 63 pages, arXiv:1307.1679.

[35] Joung E., Mourad J., Noui K., Three dimensional quantum geometry and deformed symmetry, J. Math. Phys. 50 (2009), 052503, 29 pages, arXiv:0806.4121.

[36] Kamiński W., Steinhaus S., Coherent states, $6 j$ symbols and properties of the next to leading order asymptotic expansions, J. Math. Phys. 54 (2013), 121703, 58 pages, arXiv:1307.5432.

[37] Kawamoto N., Nielsen H.B., Lattice gauge gravity, Phys. Rev. D 43 (1991), 1150-1156.

[38] Magliaro E., Perini C., Regge gravity from spinfoams, Internat. J. Modern Phys. D 22 (2013), 1350001, 21 pages, arXiv:1105.0216.

[39] Majid S., Schroers B.J., q-deformation and semidualization in 3D quantum gravity, J. Phys. A: Math. Gen. 42 (2009), 425402, 40 pages, arXiv:0806.2587.

[40] Mizoguchi S., Tada T., Three-dimensional gravity from the Turaev-Viro invariant, Phys. Rev. Lett. 68 (1992), 1795-1798, hep-th/9110057.

[41] Noui K., Perez A., Three-dimensional loop quantum gravity: physical scalar product and spin-foam models, Classical Quantum Gravity 22 (2005), 1739-1761, gr-qc/0402110. 
[42] Noui K., Perez A., Pranzetti D., Canonical quantization of non-commutative holonomies in $2+1$ loop quantum gravity, J. High Energy Phys. 2011 (2011), no. 10, 036, 21 pages, arXiv:1105.0439.

[43] Noui K., Perez A., Pranzetti D., Non-commutative holonomies in $2+1$ LQG and Kauffman's brackets, J. Phys. Conf. Ser. 360 (2012), 012040, 4 pages, arXiv:1112.1825.

[44] Oriti D., The microscopic dynamics of quantum space as a group field theory, in Foundations of Space and Time: Reflections on Quantum Gravity, Editors J. Murugan, A. Weltman, G. Ellis, Cambridge University Press, Cambridge, 2012, 257-320, arXiv:1110.5606.

[45] Oriti D., Raasakka M., Quantum mechanics on SO(3) via non-commutative dual variables, Phys. Rev. D 84 (2011), 025003, 18 pages, arXiv:1103.2098.

[46] Perelomov A., Generalized coherent states and their applications, Texts and Monographs in Physics, Springer-Verlag, Berlin, 1986.

[47] Perez A., The new spin foam models and quantum gravity, Papers Phys. 4 (2012), 040004, 37 pages, arXiv:1205.0911.

[48] Perez A., The spin foam approach to quantum gravity, Living Rev. Relativ. 16 (2013), 3, 128 pages, arXiv:1205.2019.

[49] Ponzano G., Regge T., Semiclassical limit of Racah coefficients, in Spectroscopy and Group Theoretical Methods in Physics, Editor F. Block, North Holland, Amsterdam, 1968, 1-58.

[50] Pranzetti D., Turaev-Viro amplitudes from $2+1$ loop quantum gravity, Phys. Rev. D 89 (2014), 084058, 14 pages, arXiv:1402.2384.

[51] Regge T., Williams R.M., Discrete structures in gravity, J. Math. Phys. 41 (2000), 3964-3984, gr-qc/0012035.

[52] Reisenberger M.P., Rovelli C., "Sum over surfaces" form of loop quantum gravity, Phys. Rev. D 56 (1997), 3490-3508, gr-qc/9612035.

[53] Reshetikhin N., Turaev V.G., Invariants of 3-manifolds via link polynomials and quantum groups, Invent. Math. 103 (1991), 547-597.

[54] Rovelli C., Basis of the Ponzano-Regge-Turaev-Viro-Ooguri quantum-gravity model is the loop representation basis, Phys. Rev. D 48 (1993), 2702-2707, hep-th/9304164.

[55] Rovelli C., Quantum gravity, Cambridge Monographs on Mathematical Physics, Cambridge University Press, Cambridge, 2004.

[56] Sahlmann H., Thiemann T., Chern-Simons theory, Stokes' theorem, and the Duflo map, J. Geom. Phys. 61 (2011), 1104-1121, arXiv:1101.1690.

[57] Sahlmann H., Thiemann T., Chern-Simons expectation values and quantum horizons from loop quantum gravity and the Duflo map, Phys. Rev. Lett. 108 (2012), 111303, 5 pages, arXiv:1109.5793.

[58] Schroers B.J., Combinatorial quantization of Euclidean gravity in three dimensions, in Quantization of Singular Symplectic Quotients, Progr. Math., Vol. 198, Editors N. Landsman, M. Pflaum, M. Schlichenmaier, Birkhäuser, Basel, 2001, 307-327, math.QA/0006228.

[59] Sengupta A.N., The volume measure for flat connections as limit of the Yang-Mills measure, J. Geom. Phys. 47 (2003), 398-426.

[60] Thiemann T., Modern canonical quantum general relativity, Cambridge Monographs on Mathematical Physics, Cambridge University Press, Cambridge, 2007.

[61] Turaev V.G., Viro O.Y., State sum invariants of 3-manifolds and quantum 6j-symbols, Topology 31 (1992), 865-902.

[62] Witten E., Quantum field theory and the Jones polynomial, Comm. Math. Phys. 121 (1989), 351-399.

[63] Witten E., On quantum gauge theories in two dimensions, Comm. Math. Phys. 141 (1991), 153-209. 